Originalveröffentlichung in: Dietrich Boschung, Patric A. Kreuz, Tobias Kienlin (Hg.), Biography of Objects. Aspekte eines kulturhistorischen Konzepts. (Morphomata 31) München 2015, S. 87-123

KERSTIN P. HOFMANN

\title{
IN GESCHICHTEN VERSTRICKT ... MENSCHEN, DINGE, IDENTITÄTEN
}

\begin{abstract}
„Der Zusammenhang zwischen den Wozudingen und den Geschichten ist nicht schwer zu erkennen. Man könnte diesen zunächst dahin festlegen, daß jedes Wozuding gleichsam auch eine Geschichte hat. “2
\end{abstract}

Bereits im Jahr 1953 formulierte der Geschichtenphilosoph Wilhelm Schapp in seinem Werk „In Geschichten verstrickt. Zum Sein von Menschen und Dingen" einen der radikalsten, aber bisher in den Kulturwissenschaften

1 Der Artikel baut auf meinen Arbeiten als Nachwuchsgruppenleiterin der key topic group „Identities. Space and Knowledge Related Identification“ des Berliner Exzellenzclusters 264 ,TOPOI - The Formation and Transformation of Space and Knowledge in Ancient Civilizations' auf, dem ich für die Finanzierung und das inspirierende Forschungsumfeld danke. Insbesondere die Organisation einer von der Arbeitsgemeinschaft Theorien in der Archäologie e. V. (AG TidA) in Kooperation mit den Exzellenzclustern TOPOI und „Asia and Europe in a global context“ (Heidelberg) durchgeführten Tagung zu „Massendinghaltung in der Archäologie. Der material turn und die Ur- und Frühgeschichte“, 23--25. Mai 2013 und die Mitherausgabe des Tagungsband waren hier prägend; vgl. Hofmann u.a. im Druck. Für Hinweise und kritische Anmerkungen gilt mein Dank den Teilnehmern und Teilnehmerinnen der Veranstaltungen in Berlin und Köln sowie Matthias Jung, Sabine Pinter und Stefan Schreiber. Ganz besonders möchte ich mich bei Felix Wiedemann bedanken, der mich auf Schapps Geschichtenphilosophie aufmerksam machte und mich bei der Einarbeitung in die Erzähltheorie unterstützte. Die Verantwortung für den Inhalt und vor allem etwaige Fehler liegt trotz dieser vielfältigen Unterstützung selbstverständlich jedoch allein bei mir.

2 Schapp 1953, 3. 
kaum rezipierten Ansätze, das gemeinsame In-der-Welt-Sein von Dingen und Menschen als Geschichten zu interpretieren. Er ging dabei aber einen ganz anderen Weg als viele der heutigen sogenannten Objektbiographien bzw. life histories, denn weder das gesamte Leben eines Einzelobjektes, noch die Frage der agency wird von ihm behandelt. Es stellen sich daher folgende Fragen: Wie können von uns heute geborgene Funde und Befunde als Handlungsträger/-innen in wissenschaftlichen Erzählungen eingebettet werden? Können wir in der Prähistorischen Archäologie wirklich Biographien mit archäologischen Funden und Befunden als Protagonist/-innen schreiben und welche Implikationen hat dieser immer häufiger geäußerte Wunsch? Und last but not least welche Alternativen, Ding- bzw. Objektgeschichten zu verfassen, gibt es?

Für die Prähistorische Archäologie sind Untersuchungen von an Funden materialisierten Transformationen und die vergleichende Analyse von Funden und Befunden die einzigen Wege, Veränderungen und Wandel an Dingen bzw. Objekten für die Prähistorie nachzuvollziehen. Ergänzende Textquellen, teilnehmende Beobachtungen oder Befragungen sind nicht möglich. Und so müssen prähistorische Archäologen und Archäologinnen, wenn sie Objektbiographien schreiben wollen, allein mit ihren eigenen Verstrickungen und den im archäologischen Befund überlieferten materiellen Überresten eines Objektes auf dessen ,Leben' rückschließen. Während der ,Tod' des Objektes im archäologischen Befund noch vergleichsweise gut zu fassen ist und die, Geburt' u. a. durch Form und Material - oft jedoch nur verallgemeinernd - (re)konstruiert werden kann, ist das Leben des als kontinuierlich existierend angenommenen Objektes in seiner Dauer und seinen Stationen - wenn überhaupt - nur schwer durch Gebrauchsspuren, Transformationen und Rekontextualisierungen einzuschätzen. Die erste Begeisterung, Dinge bzw. Objekte - also genau das, was wir in der Prähistorischen Archäologie im Überfluss haben und in dessen Beschreibung und Analyse wir von den anderen Kulturwissenschaften anerkannt eine besondere Expertise besitzen - zu Protagonist/ -innen von Geschichten zu machen, ist nur zu verständlich ${ }^{3}$. Aufgrund unserer Quellenlage und den von uns untersuchten Zeitdimensionen mit geringer Auflösung verfassen wir meist jedoch andere Geschichten mit Dingen oder Objekten, als sie in den Kultur- und Sozialwissenschaften

3 Exemplarisch seien hier nur einige wenige der inzwischen zahlreich erschienenen Arbeiten mit ,objektbiographischem'Ansatz genannt: Gosden/ Marshall 1999. - Holtorf 2000-2008. - Fontijn 2002. - Joy 2009. - Kistler 2010 . 
seit Igor Kopytoffs ${ }^{4}$ Einführung der Metapher „Objektbiographie“ gefordert werden. Dies ist grundsätzlich nicht problematisch, weil Metaphern in der Wissenschaft vor allem die Kreativität anregen und neue Untersuchungsfelder erschließen sollen ${ }^{5}$ und die Übersetzung von Konzepten in andere Zusammenhänge immer dringend notwendig ist ${ }^{6}$. Die Gefahr des Wiederbelebens eines gerade in Ansätzen überwunden geschienenen naiven Materialismus ist hier jedoch aufgrund des schon immer vorhandenen methodologischen Fetischismus der Prähistorischen Archäologie gegeben. Neben der drohenden Authentizitätsfalle, d.h. der Versuchung anzunehmen, dass man durch Gewahrwerden ,authentischer Substanz einen direkten Zugang zur Geschichte hätte ${ }^{7}$, geht der Ansatz zudem oft mit einer erneuten Aufwertung außergewöhnlicher Einzelfunde und einer Darstellung kausaler Linearität einher.

Im Folgenden soll daher versucht werden, durch theoretisch-methodische Reflexionen die Verstrickung von Menschen, Dingen und Identitäten in Geschichten zu beleuchten und dabei Perspektiven, aber auch Risiken aufzuzeigen, die die verschiedenen Ansätze, Ding- oder Objektgeschichten zu schreiben, mit sich bringen. Beginnen möchte ich mit einigen Überlegungen zum Begriff Geschichte bzw. Geschichten und zum Verhältnis von Leben und Erzählen. Anschließend werden unter der Überschrift „Archäologischer (Be)Fund: Ding, Objekt und Subjekt“ die potentiellen Handlungsträger/-innen archäologischer Erzählungen und der methodologische Zugang zu ihnen behandelt. Diese Vorbemerkungen erlauben es dann, verschiedene Formen von Ding- bzw. Objektgeschichten näher zu charakterisieren. Ausführlicher soll dabei die Geschichtenphilosophie Schapps und seine Wozudinge vorgestellt werden; nicht als Alternative, sondern als komplementärer Ansatz. In der Synthese möchte ich abschließend zwei Fragen behandeln: Erstens, ob die Metapher der Objektbiographie für die Prähistorische Archäologie gewinnbringend war und ist und zweitens, ob es eine Entwicklung von History zu itstories ${ }^{8}$ gibt bzw. geben sollte.

4 Kopytoff 1986.

5 Siehe Finke 2013.

6 Bachmann-Medick 2014.

7 Siehe z. B. Knigge 2011. - Saupe 2014.

8 Wortspiel in Anlehnung an die Gegenreaktionen auf die lange Zeit überwiegend geschriebene Ereignisgeschichte großer Männer in Form einer Frauengeschichte (herstory) und einer "Geschichte von unten“ (history from below) mit Bezug auf den in den Literaturwissenschaften geprägten Begriff der it-narratives für Geschichten, die vor allem in der 2. Hälfte des 18. Jhs. 


\section{GESCHICHTE(N): ZUM VERHÄLTNIS VON LEBEN UND ERZÄHLEN}

Die Geschichte im allgemeinen Sinne ist alles, was geschehen ist, und wird daher mitunter auch synonym mit Vergangenheit verwendet. Daneben steht der Begriff ,Geschichte' aber auch für die Betrachtung der Vergangenheit im Gedenken, im Erzählen und in der Geschichtsschreibung. Zudem kann Geschichte auch eine Handlung oder Erzählung sein. Unter Geschichte bzw. Geschichten wird demnach sowohl Referenz, Praxis als auch das Produkt des Erzählens und seine Rezeption verstanden ${ }^{10}$ (Abb. 1).

Erzählen wird dabei oft als universelle Fähigkeit des Menschen aufgefass $\mathrm{t}^{11}$. Erzählungen und ihre Erforschung haben jedoch verschiedene Konjunkturen durchlaufen ${ }^{12}$. Nach Kritik durch den Poststrukturalismus und trotz oder gar wegen des Zweifels an den ,grand narratives' haben Erzählungen heute wieder Hochkonjunktur: Sei es im Plural als postmoderne Antwort auf den Verlust des Glaubens, eindeutige Wahrheit und Wissen erlangen zu können, oder im Singular als Weg, kognitive

in Großbritannien aus Perspektive der eines Objektes geschrieben wurden; siehe hierzu u. a. Johansson 1976. - Paul/Schossig 1986. - Lupton 2006.

9 Für die Zurverfügungstellung zweier noch unveröffentlichter Manuskripte zum Thema Erzählen in den Altertumswissenschaften, die im Zuge des Projektes „Wanderungsnarrative und Identitätsräume“ der Forschungsgruppe B-4 „Space - Identity - Locality“ des Berliner Exzellenzclusters Topoi“ und des DFG-geförderten Projektes „Wanderungsnarrative in den Wissenschaften vom Alten Orient (1870-1930)“ entstanden sind, möchte ich mich an dieser Stelle bei Felix Wiedemann bedanken; siehe Saupe/ Wiedemann 2015. - Cancik-Kirschbaum/Wiedemann in Vorbereitung.

10 Vgl. Koselleck 1979, 48-49. 130-143. - Goetz 1993, 16-21. - Fludernik 2010. Der Zusammenhang von Forschen und Erzählen wurde vor allem in den Disziplinen Geschichte (siehe z. B. White 1986. - White 1990. - Rüsen 1990. - Jaeger 2002) und Ethnologie (siehe z. B. Bruner 1986. - Kohl 1992. Geertz 1993) bereits früh thematisiert, in den letzten Jahrzehnten ist jedoch auch von archäologischer Seite ein zunehmendes Interesse feststellbar; siehe z. B. Pluciennik 1999. - Jackman/Witmore 2002. - Leskovar 2005. Veit 2006. - Rieckhoff u. a. 2010.

11 So z. B. bei Roland Barthes $(1988,102)$ mit seinem universalistischen Erzählbegriff: „sie [die Erzählung] ist international, transhistorisch, transkulturell, und damit einfach da, so wie das Leben."; siehe ferner Koschorke 2012, 9-12 und Straub 2013, die vom homo narrans bzw. homo narrator sprechen.

12 Siehe Nünning 2012. 


\begin{tabular}{|c|c|}
\hline Vergangenheit & $\begin{array}{c}\text { die Geschichte } \\
\text { im Kollektivsingular } \\
\text { (vgl. Koselleck 1979; 48-49. 130-143) }\end{array}$ \\
\hline $\begin{array}{l}\text { historische Geschehen } \\
\text { (Ereignisse und Begebenheiten) } \\
\text { mit historischen Akteuren }\end{array}$ & $\begin{array}{c}\text { Geschichten } \\
\text { im Sinne von Schapp } 1953\end{array}$ \\
\hline $\begin{array}{c}\text { Darstellungen } \\
\text { mit Figuren/Charakteren/(historiographischen) } \\
\text { Akteuren, die im Sinne Greimas Aktanten-Modell } \\
\text { oft bestimmte Funktionen bzw. Handlungsrollen } \\
\text { in Darstellungen einnehmen, sowie Handlungen } \\
\text { und Settings }\end{array}$ & $\begin{array}{l}\text { Geschichte(n) } \\
\text { als Darstellungen (u. a. Erzăhlungen); } \\
\text { hier vor allem im Sinne der Geschichtsschreibung } \\
\text { als Rekonstruktionen und Reprösentotionen } \\
\text { historischen Geschehens relevont }\end{array}$ \\
\hline
\end{tabular}

1 Geschichte(n). Versuch einer operationalen Begriffsklärung

\begin{tabular}{|l|l|l|}
\hline $\begin{array}{l}\text { Phänomenologie \& } \\
\text { Philosophische Hermeneutik } \\
\text { „narratives in-der-Welt-Sein" }\end{array}$ & Leben -> Erzăhlen & $\begin{array}{l}\text { 2. B. Wilhelm Dilthey, } \\
\text { Hannah Arendt, David Carr, } \\
\text { Alisdair Macintyre }\end{array}$ \\
\cline { 2 - 3 } & Welt = Geschichten & Wilhelm Schapp \\
\hline $\begin{array}{l}\text { narrativer Konstruktivismus } \\
\text { (Bruch, Dualismus) }\end{array}$ & Leben | Erzăhlen & $\begin{array}{l}\text { 2. B. Louis Mink, Hayden } \\
\text { White, } \\
\text { Frank Ankersmit }\end{array}$ \\
\hline $\begin{array}{l}\text { "Synthesis des Heterogenen" } \\
\text { (Mimesis: Prã-, Kon-, Refiguration) }\end{array}$ & Leben <-|-> Erzăhlen & Paul Ricceur \\
\hline
\end{tabular}

2 Zum Verhältnis von ,Leben' (bzw. ,Geschehen') und ,Denken“ (bzw. ,Erzählen') (Zitate: Ricœr 2007a, 218 und 106)

Prozesse des Menschen besser verstehen zu können ${ }^{13}$, bzw. im Rahmen neuer Metanarrative, welche die zunehmende Komplexität der Welt durch menschliche Wesensmerkmale zu erklären versuchen ${ }^{14}$.

Die soeben geschilderte Gemengelage erfordert es, sich mit den verschiedenen theoretischen Positionen zum Verhältnis von ,Leben' bzw. Geschehen und Denken bzw. Erzählen auseinanderzusetzen (Abb. 2). Vor allem in der Phänomenologie, aber auch in der philosophischen Hermeneutik werden der narrative Charakter des Lebens und die Kontinuität

13 Ryan 2007, 22.

14 In der Prähistorischen Archäologie sprach sich Andrew Sherrat bereits 1995 für ein Wiederbeleben von Metanarrativen aus (Sherratt 1995), die jedoch eigentlich nie ganz verschwunden waren (siehe Veit 2010, 18-23). Aktuelle Beispiele sind die entanglement-Theorie Ian Hodders (2012; siehe auch Pollock u. a. in Review) und John Chapmans fragmentation-Ansatz (siehe Chapman 2000. - Chapman/Gaydarska 2009). 
zwischen Leben und Erzählen betont ${ }^{15}$. „Geschichten werden gelebt, bevor sie erzählt werden - außer in Romanen", so z. B. der schottisch-amerikanische Philosoph Alisdair MacIntyre ${ }^{16}$. Für Schapp ist sogar ein (Da-)Sein außerhalb von Geschichten nicht möglich. Die Welt und die Geschichten, in die wir verstrickt sind, fallen zusammen ${ }^{17}$. Eine dualistische Auffassung vertreten dahingegen die Anhänger/-innen des narrativen Konstruktivismus, zwischen Leben und Erzählen gibt es nach ihnen einen Bruch ${ }^{18}$. Der amerikanische Geschichtsphilosoph Louis Mink betonte z. B. „stories are not lived but told“19. Paul Ricœur entwickelte in seinem dreibändigen Opus „Zeit und Erzählung“ eine vermittelnde Position, indem er durch den „Kreis der Mimesis“ bestehend aus Präfiguration, Konfiguration und Refiguration das Vorher und Nachher der Erzählung und damit Referenz und Rezeption mitberücksichtigt ${ }^{20}$. Mimesis, und insbesondere die Mimesis II der Konfiguration, wird nicht als einfache Kopie, sondern als schöpferischer Akt verstanden. Leben und Erzählen bleiben somit unterscheidbar, stehen aber in einer engen Wechselbeziehung.

Die Begriffsklärung, was nun konkret unter einer Erzählung zu verstehen sei, erweist sich aufgrund der vielfältigen und $z$. T. widersprüchlichen Verwendung des Wortes als schwierig ${ }^{21}$. Als operationale Minimaldefinition schlage ich in diesem Zusammenhang vor, faktuale Erzählungen ${ }^{22}$ als Repräsentationen eines Geschehens aufzufassen, welches - z. T. unerwartete - Veränderungen bewirkt, somit Konsequenzen hat und von Bedeutung ist. In Erzählungen werden individualisierte Figuren, Handlungen und Settings im Rahmen einer mehr oder minder fixierten sequentiellen temporalen Struktur aufeinander bezogen. Erzählungen überführen so einen Anfangsstatus sinnvoll bzw. nachvollziehbar in einen Endzustand. Sie anerkennen und bewältigen zugleich Kontingenz ${ }^{23}$.

15 Siehe u. a. Dilthey 1927. - Arendt 2011. Kritik am später aufkommenden narrativen Konstruktivismus äußerte u.a. der Phänomenologe und Husserl-Forscher David Carr mit seiner These einer Kontinuität von Leben und Erzählen; Carr 1986.

16 MacIntyre 1995, 283.

17 Schapp 1953, 1.

18 z. B. Ankersmit 1983. - White 1990.

19 Mink 1970, 557.

20 Ricœur 2007a, 87-135; siehe ferner Meuter 1995, 122-175.

21 Siehe Ryan 2007, 23-24. - Nünning 2012.

22 Siehe Klein/Martinéz 2009.

23 Ricœur 1986, 16. - Ricœur 1996, 176; siehe ferner Müller/Rüsen 1997. 
Unter Biographien, eine Spezialform der Erzählung, die an der Schnittstelle zwischen Literatur-, Geschichts- und Kulturwissenschaft angesiedelt ist, werden traditionell evidenznahe Repräsentationen vergangenen Lebens eines - oft in irgendeiner Form ,bedeutenden ' - Individuums innerhalb spezifischer struktureller Zusammenhänge verstanden ${ }^{24}$. Anfang und Ende sind klassischer Weise durch Geburt und Tod vorgegeben. Wobei durch den Wortbestandteil graphus auch das Einschreiben von Bedeutung thematisiert ist. Als biographische Kennmarken gelten heute Identität, Individualität, Vergesellschaftung und Handlungsfreiheit ${ }^{25}$.

Ein festes Element von Erzählungen ist der Plot bzw. das Narrativ. Den Unterschied zwischen der Wiedergabe aneinandergereihter Ereignisse, in der Narratologie auch als Handlung oder Geschichte tituliert, und einem Plot verdeutlichte bereits der englische Literat Edward Morgan Forster (1879-1970) anhand zweier Aussagen sehr eindrücklich: „Der König starb und dann starb die Königin“ ist eine Handlungswiedergabe, die nur auf die Frage „und dann?“ antwortet; „Der König starb und dann starb die Königin aus Kummer“ ist eine Erzählung, die der Frage „warum?“ - und zwar in einem konkreten Einzelfall - gerecht wird ${ }^{26}$.

Dies leitet über zu der Frage, wie sich Erzählungen von anderen Texttypen bzw. Darstellungsformen unterscheiden. Als Gegenpole werden oft die Argumentation und die Beschreibung ausgemacht ${ }^{27}$. Während erstere - meist paradigmatisch - überzeugen will, schafft letztere Ordnung durch wiedergegebene Kriterien. Faktuale Erzählungen bewegen sich oft zwischen diesen beiden Polen. Sie sind weder generalisierend, noch konkret, auf einen Status zeitlich fixiert, sondern individualisierend, zugleich aber verknüpfend Veränderungen darstellend. Sowohl Argumentation als

24 Vgl. Fetz 2009, 5-10. 39. Das Genre ist jedoch äußerst heterogen und gerade seit dem 20. Jh. gibt es z. B. viele vom Ideal der linearen Heldenbiographie abweichende ,Anti-Biographien'. Ferner gibt es in der Historiographie schon früh organologische Beschreibungen z. B. des Aufstiegs, der Blüte und des Verfalls von Reichen oder Städten, die man im weitesten Sinne auch als Biographien bezeichnen könnte. Referenz ist hier wie später in der Ethnologie und Archäologie jedoch - obwohl selten expliziert - die klassische Form der Biographie. Allerdings wirken natürlich auch diese Adaptionen rück bzw. es wird sich in Fachkreisen auf diese statt auf die klassischen Biographien bezogen.

25 Fetz 2009, 11.

26 Forster 1985, 86.

27 z. B. Chatman 1990; siehe auch Ryan 2007, 26-27. - Kleemann u. a. 2007, 65-66. 
auch Erzählung bewältigen Kontingenz und erklären. Erstere aber durch die Zurückführung des Neuen auf das Alte bzw. des Verschiedenen auf das Immergleiche, letztere durch die genaue Beschreibung, die die Aufmerksamkeit auf Sinnzusammenhänge, Übergänge und Plausiblitäten lenkt ${ }^{28}$. Nicht nur für die Geschichtswissenschaft besteht dabei jedoch stets die Gefahr eines „story bias“29, d. h. Sachverhalte durch narrative Sinnzuweisungen zu verdrehen bzw. zu vereinfachen und unreflektiert überkommene narrative Strategien und Muster anzuwenden ${ }^{30}$.

$\mathrm{Zu}$ jeder Erzählung gehören ferner Figuren oder Charaktere, die als Handlungsträger/-innen bzw. nach dem Strukturalisten und Textsemiotiker Algidar Julien Greimas als Aktanten fungieren (Abb. 1) ${ }^{31}$. Im Unterschied zu Akteuren, die konkrete Figuren sind, in denen sich Rollen verkörpern, spricht Greimas von Aktanten, wenn er die von ihm herausgearbeiteten, zeitlosen abstrakten Bedeutungseinheiten meint, die als funktionale Träger von Aktionen auftreten. Der Begriff wurde später von Bruno Latour für seine Akteur-Netzwerk-Theorie aufgegriffen, da man in der Soziologie alles wie eine Erzählung analysieren könne und es auch hier Einheiten gäbe, die tragende Rolle einnähmen bzw. die Ursachen von Wirkungen seien ${ }^{32}$. Allerdings transformieren bei Latour die Aktanten, bei denen es sich nach ihm nicht nur um menschliche Wesen, sondern um alle Arten von vernetzten Handlungseinheiten aus Lebewesen, Objekten und Konzepten handeln kann, zu Akteuren ${ }^{33}$. Welche Rolle spielen archäologische (Be)Funde nun aber in bzw. für archäologische(n) Erzählungen und wie werden die historiographischen Akteure ermittelt und bezeichnet?

28 Hampe 2007, 22-28; siehe ferner Ricœur 2007a, 181-262.

29 Dobelli 2011, 53-56.

30 Kritisch zu (unreflektierten) archäologischen Erzählungen äußerten sich u. a. Eggert 2006, 218. - Jung 2010. Zur langen Tradition von Herkunftsund Wanderungsnarrative siehe Wiedemann 2010. - Wiedemann u.a. in Vorbereitung. Reinhard Bernbeck (2013) plädierte kürzlich in Anlehnung an den noveau roman für ein subjektloses Erzählen. An deren konkreten Umsetzung fehlt es allerdings noch, so dass eine endgültige Beurteilung dieses Versuchs, neuere Erzählformen für die Archäologie zu erschließen, noch nicht erfolgen kann. Allerdings dürfte es m. E. schwierig sein, Vergangenheit gänzlich subjektlos über emotional geprägte Erfahrungen - in seinen Beispielen Eifersucht und existenzielle Not - vermitteln zu wollen. 31 Greimas 1971; siehe hierzu ferner Ricœur 2007b, 78-103. - Kim 2002.

32 Ruffing 2009, 35-36. - Schüttpelz 2013, 19-25.

33 Vötsch 2010, 38. 


\subsection{DINGE UND OBJEKTE: EINE BEGRIFFSKLÄRUNG}

Verwendete man vor einigen Jahren in der Archäologie die Begriffe Artefakt und Objekt noch weitgehend synonym und vermied das Wort ,Ding - galt dies doch als eine umgangssprachliche, herabsetzende Bezeichnung für gerade nicht näher bestimmte Gegenstände -, kommt man heute im Rahmen von Arbeiten zur materiellen Kultur kaum mehr um eine Unterscheidung dieser Begriffe herum (Abb. 3).

Hier sei ,Ding' als Oberbegriff für Entitäten verstanden, die im Rahmen von Handlungen wahrnehmbar, aber in ihrem, in der Welt sein' nur sporadisch - meist wenn sie gerade ihren Zweck nicht erfüllen oder stören - ins Bewusstsein vordringen. Sie zeichnen sich durch ihre physische Erscheinungsform, sprich ihre Materialität, Dreidimensionalität und physische Präsenz aus ${ }^{34}$. Grundsätzlich würden nach dieser Definition sowohl archäologische Funde als auch Befunde unter den Begriff Ding fallen können, meist wird er aber nur für Artefakte, sprich mobile, vom Menschen produzierte Gegenstände, verwendet.

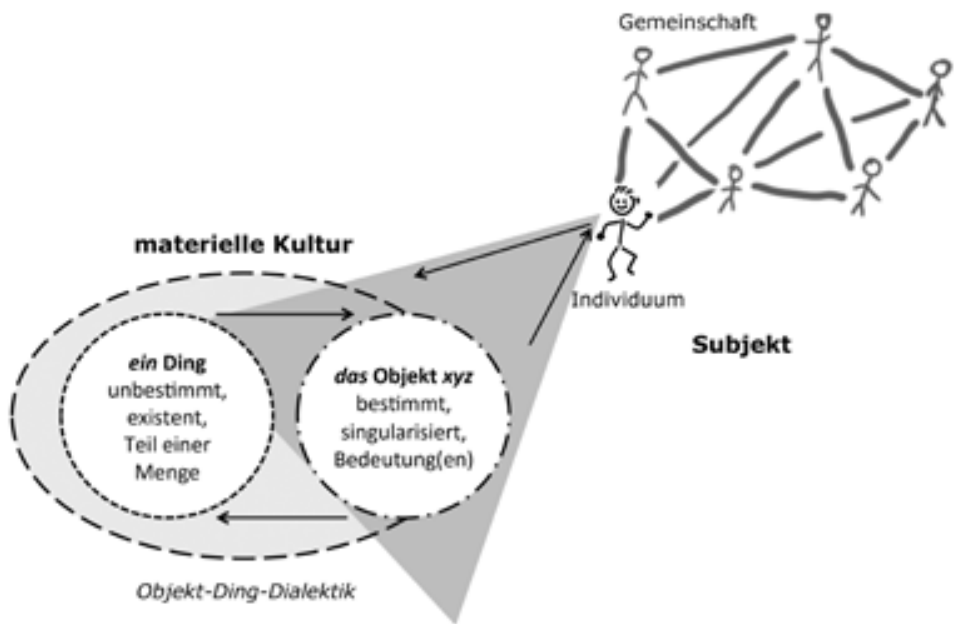

3 Zum Verhältnis von Dingen, Objekten und Subjekten

34 Vgl. Pearce 1993, 4-5. 15-35. 
Die Bedeutung, die wir Dingen z. B. schon durch den Vorgang der Kategorisierung beimessen, macht sie zu Objekten ${ }^{35}$. Durch Wahrnehmungshandeln ${ }^{36}$ eines Subjektes kann so aus einem undefinierten, existenten Ding das Objekt $x y z$ mit spezifischen, allerdings veränderbaren und mehrwertigen Bedeutungen werden ${ }^{37}$. Seine Konkretheit und Identifizierbarkeit sind letztlich Bedingung dafür, dass das Objekt Zeichencharakter erhält und für ein anderes als es selbst stehen kann ${ }^{38}$.

Diese Bestimmung weicht von Definitionen ab, die dem Objekt gegenüber dem Subjekt eine Eigenständigkeit zuschreiben und es in einer ,objektiven Welt' als unabhängig von den Beobachter/-innen existent ansehen. Nach obiger Definition wäre dies, wenn überhaupt, der stets mitexistierende und -wirkende Ding-Anteil des Objektes. Wobei jedoch einschränkend zugestanden werden muss, dass auch das Ding hier bereits als aus der Außenwelt hervorgehoben und kulturell definiert ist. Die Formulierung „In der Erkenntnis bestimmt das (erscheinende) Objekt das Subjekt, in der Handlung hingegen macht das Subjekt das Objekt zum

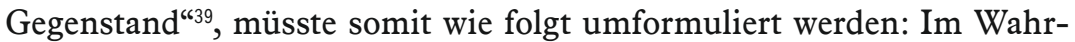
nehmungshandeln macht das Subjekt kulturell (vor)definierte Dinge zu Objekten, die wiederum die Erkenntnisse des Subjektes beeinflussen. So lässt das Objekt stets das Subjekt und das Subjekt das Objekt mitdenken. Interessant ist hier zudem die Entwicklung in der Kunst von Objekten

35 Ludwig 2011, 2.

36 Statt Wahrnehmung allein auf die Fähigkeiten unserer Sinnesorgane oder die objektive Existenz einer Außenwelt zu reduzieren und diese als vorgängig anzusehen, wird durch das Konzept des Wahrnehmungshandelns betont, dass wir die Qualitäten eines Dinges nur durch Handeln in einer Umgebung erfahren und erzeugen, in der Dinge und Mensch eingebettet sind. Handeln ist zugleich Wahrnehmen und andersherum, es ist stets leiblich und synästhetisch, d. h. es läuft über den zeitlich-praktischen Zusammenhang aller Sinne; Frers 2009, 188.

37 Siehe auch Ivleva im Druck, Abb. 1. Dies widerspricht scheinbar zwar der Aussage Jacques Derridas, dass ein Ding kein Objekt ist und es auch keines werden kann; Derrida 1984, 14. Allerdings nur dann, wenn das Objekt zum reinen Zeichen bzw. zur Idee würde und somit nicht mehr Teil der materiellen Kultur wäre und gänzlich seinen Ding-Charakter verlöre. Dinge werden jedoch auch hier nicht als vollständig in die Kategorie Objekt transformierbar angesehen, sondern behalten mit Hans-Peter Hahn stets ihren „Eigensinn“; Hahn 2011. - Hahn 2013; siehe ferner Brown 2001.

$38 \mathrm{Kohl}$ 2003, 120.

39 Hartmann 1962, 100. 
hin zu emanzipierten Dingen, wie sie vor allem in der Konzeptkunst anzutreffen ist ${ }^{40}$. Nur eine - in diesem Zusammenhang allerdings wichtige Randnotiz: Kopytoff unterschied in seinem bahnbrechenden Artikel "The cultural biography of things" nicht systematisch zwischen ,things' und ,objects', sondern nur zwischen kommodifizierten und singularisierten Dingen bzw. Objekten ${ }^{41}$. Im Folgenden soll, jedenfalls wenn es um materielle Kultur geht, mit Andreas Ludwig „von ,Dingen“ immer dann gesprochen [werden / K. H.], wenn es um ihr reines Vorhandensein, ihren Gebrauchswert oder den historischen Nutzungskontext geht, von ,Objekten' [bei denen es sich immer auch um Dinge handelt / K. H.] aber in Zusammenhang mit ihrer kulturellen Codierung und historischen Interpretation. ${ }^{\star 42}$.

\subsection{ARCHÄOLOGISCHE (BE)FUNDE: DINGE ODER OBJEKTE?}

Geht man nun vom archäologischen Befund aus, könnte man sich die Frage stellen, ob die jeweils wahrgenommene und dokumentierte Entität zum Zeitpunkt der Einlagerung in den Boden ein Ding oder ein Objekt war $^{43}$. Den meisten Grabbeigaben wird gewöhnlich ein biographischer Objektcharakter zugesprochen bzw. es wird für sie zumindest eine kulturelle Codierung angenommen ${ }^{44}$. Hier könnte man versuchen, mit der Unterscheidung der französischen Soziologin Violette Morin zwischen biographischem, persönlichem und protokollarischem, welt-gewandtem Objekt ${ }^{45} \mathrm{zu}$ operieren, um mögliche voreilige Schlüsse über die Identität der Toten zu vermeiden. Auch für sogenannte Weihefunde wird zumeist ein Objektcharakter angenommen, während es sich bei Verwahrfunden sowohl um Waren als auch um singularisierte Objekte handeln kann. Die Keramikscherbe eines Gefäßes, geborgen in einer aufgelassenen Siedlung wird indes, wenn überhaupt, wahrscheinlich nur noch Ding-Charakter gehabt haben. Noch schwieriger ist es, Befunde, wie z. B. Grabhügel, zu beurteilen. Unmittelbar anschließend an seine Errichtung hatte dieser wohl für die Bestattungsgemeinschaft aufgrund der Erinnerung an den Bau und die Bestattung eher Objektcharakter. Später war er zwar eventuell sichtbar, ob er jedoch als Ding wahrgenommen wurde oder gar im

40 Vgl. Brown 2001. - Rübel 2009. - Rieckhoff im Druck.

41 Kopytoff 1986.

42 Ludwig 2011, 3.

43 Siehe auch Fontijn 2002, 33.

44 Siehe Whitley 2002.

45 Morin 1969, 132-133. 
Wahrnehmungshandeln stets eine Rolle als Objekt gespielt hat, ist heute meist schwerlich feststellbar. Zumindest Nachbestattungen und etwaige Denominationen, wie z. B. Galgenberg, deuten jedoch darauf hin, dass sie immer wieder zu Objekten werden konnten.

\subsection{ZUR GRENZZIEHUNG ZWISCHEN MENSCHEN \\ UND DINGEN UND DER METHODOLOGISCHE FETISCHISMUS \\ DER PRÄHISTORISCHEN ARCHÄOLOGIE}

Alle archäologischen (Be)Funde können als Dinge oder Objekte in Darstellungen theoretisch Handlungsträger/-innen sein, und zwar ohne dass man ihnen auch in der Vergangenheit zugleich eine Handlungsfähigkeit und Intentionalität zuschreiben müsste. Allerdings steht nach Albrecht Koschorke das Erzählen „im Bund mit einem Animismus, der alle Wesen beseelt und mit Handlungsmacht ausstattet“ und selbst „uneinheitliche, unpersönliche Vorgänge [würden / K. H.] durch erzählerische Bearbeitung den Charakter eines sinnhaften personalisierten Geschehens ${ }^{\star 46}$ annehmen. Insbesondere im Zusammenhang mit Objektbiographien wird daher auch immer wieder die Frage nach der Grenzziehung zwischen Menschen bzw. Personen und Dingen bzw. Objekten gestellt. So auch von Kopytoff, der anhand der Sklaverei zeigt, dass Menschen zwar mitunter nicht als Personen, sondern als Dinge oder Objekte betrachtet werden, sie aber trotz ihres latenten Warencharakters oft zumindest zeitweise singularisiert sind ${ }^{47}$. Bereits Bronisław Malinowski und Marcel Mauss machten in ihren Studien zu Tauschsystemen darauf aufmerksam, dass die Grenze zwischen Personen und Dingen kulturell variabel sind ${ }^{48}$. Dinge können in Kategorien zusammengefasst oder als Objekte singularisiert z. B. ein gender zugeschrieben bekommen ${ }^{49}$, einen eigenen Namen erhalten, mit historischen und rituellen Bedeutungen versehen werden, oder so eng mit einem Menschen assoziiert werden, dass sie stellvertretend für diesen stehen $^{50}$. Ende der 1990er Jahre wies der britische Sozialanthropologe Alfred Gell in einer Untersuchung zu Kunst darauf hin, dass diese hergestellt sei, um die Gedanken und Handlungen anderer zu beeinflussen, und somit agency besäß $\mathrm{e}^{51}$. Diese Idee wurde schnell, insbesondere in der

\footnotetext{
46 Koschorke 2012, 79.

47 Kopytoff 1986.

48 Hoskins 2006, 74.

49 Siehe Strathern 1988.

50 Siehe Weiner 1992.

51 Gell 1998.
} 
Archäologie, auch auf alle anderen Arten von Dingen übertragen ${ }^{52}$. Bereits Anfang der 1980er problematisierte Donna Haraway die Grenzziehungen zwischen Mann und Frau sowie Mensch und Maschine und nutzte die Cyborg als Figur, um dichotome Kategorisierungen zu hintergehen ${ }^{53}$. In der Archäologie fand dies jedoch erst über Bruno Latour Eingang, der mit seiner Akteur-Netzwerk-Theorie Dinge als handelnde Akteure betrachtet, die zusammen mit menschlichen Akteuren in netzwerkartigen Handlungszusammenhängen agieren. Das bekannteste Beispiel für einen derartigen Aktanten ist sicherlich seine ,Mensch-Schusswaffe', die aus dem Zusammenwirken beider entstehe, aber nicht auf eine der beiden reduziert werden könne ${ }^{54}$.

Statt die Unterschiede zwischen Menschen und Dingen hervorzuheben, wurde so die Wirkmacht von Dingen betont und analog bzw. ,symmetrisch' zu denen von Menschen untersucht. In der Archäologie wird dieser Trend, Menschen und Dinge kaum noch konzeptionell zu differenzieren, von einigen Forscher/-innen besonders vehement verfolgt ${ }^{55}$. Dies war und ist jedoch nicht ein Spezifikum objektbiographischer Arbeiten, sondern z. B. auch für Ian Hodders entanglement-Theory festzustellen ${ }^{56}$. Die Neigung, Dinge und Menschen gleichzusetzen, mag zum einen am grundsätzlichen Erkenntnisproblem liegen, individualisierte menschliche Handlungsträger/-innen und Intentionalität für prähistorische Zeiten nachzuweisen. Zum anderen haben - vielleicht auch mit ersterem Grund ursächlich verknüpft - Biometaphern und methodologische Gleichsetzung von Artefakten mit Lebewesen eine lange Tradition. Die Prähistorische Archäologie hat nämlich schon immer dem von Arjun Appadurai für die soziale Analyse von Dingen notwendig betrachteten „methodologischen Fetischismus ${ }^{\text {“57 }}$ gefrönt. So war z. B. für die Datierung

52 Siehe u. a. Dobres/Robb 2000. - Gosden 2005. - Knappett/Malafouris 2008.

53 Haraway 1995; archäologische Rezeption bei Schreiber im Druck.

54 Latour 2005; siehe ferner Belliger/Krieger 2006. - Kneer u. a. 2008. Zur Rezeption in der Sozialanthropologie und Archäologie siehe u. a. Ingold 2008. - Stockhammer 2012.

55 Siehe z. B. Gosden 2005. - Shanks 2007. - Witemore 2007. - Olsen 2010. - Olsen u. a. 2012. - Pétursdóttir 2012.

56 Hodder 2012, 219. Wenn man den (objekt)biographischen Ansatz allerdings sehr weit fassen will, könnte man auch seine Subjekte und Objekte nicht nur unidirektional verknüpfende Verflechtungsgeschichte darunter fassen.

57 Appadurai 1986, 5. 
und die Schaffung einer archäologischen Tiefenzeit im 19. Jahrhundert die Übertragung des in der Geologie und Paläontologie entwickelten Konzepts des Leitfossils entscheidend. Doch bereits Rudolf Virchow wies darauf hin, wie „unsicher die archäologischen ,Leitmuscheln ${ }^{\text {““58 }}$ seien. So ist z. B. der Nutzungszeitraum von Dingen im Vergleich zum Leben von Tieren und Pflanzen wesentlich variabler und im Gegensatz zur Annahme des Diffusionismus sind Ideen und Objekte auch an unabhängig voneinander existierenden Orten entwickelbar ${ }^{59}$. Als weitere Beispiele seien hier nur kurz die berühmte Gleichung „pots = people ${ }^{“ 60} \mathrm{bzw}$. die strukturell ähnliche Zuschreibung von männlichen Waffen und weiblichem Schmuck ${ }^{61}$ erwähnt. Hier werden Menschen bzw. deren Identitäten mit Dingen gleichgesetzt und bei deren Fund sogleich mit der Anwesenheit ersterer gerechnet. So wurden und werden leider auch heute noch immer wieder Herrschaftsansprüche legitimiert und gesellschaftliche Rollenbilder zementiert. Ein Problem dieses Ansatzes ist, dass sich die kulturelle Bedeutung eines Objektes jedoch ohne materielle Folgen für ein Ding bzw. eine Dingkategorie ändern kann. Dies leitet zur Frage der Identität eines Objektes über.

\subsection{TRANSITORISCHE OBJEKTIDENTITÄTEN}

„Die Vase ist eine Vase ist eine Vase - und sei sie auch noch so weit gereist " 62

Mit dieser vehement diskutierten Aussage problematisierte der Althistoriker Ulrich Gotter die Nachweismöglichkeiten von ,Akkulturation“ allein anhand ausgetauschter Artefakte. Allerdings ist in diesem Satz nicht berücksichtigt, dass eine Vase zwar theoretisch in Materie und Form immer bzw. lange Zeit die gleiche bleiben kann, sich ihre Objektidentitäten jedoch verändern oder einem Ding sogar gleich mehrere Objektidentitäten zugeschrieben werden können. Noch gravierender ist allerdings, dass wir mitunter noch nicht einmal wissen, ob ein archäologischer $(\mathrm{Be})$ Fund auch früher immer als Entität wahrgenommen wurde. Dies würde aber eine evidenznahe Repräsentation des Lebens eines Dings im Sinne

58 Virchow 1881, XI.

$59 \mathrm{Vgl}$. Hofmann 2014, 132.

$60 \mathrm{Vgl}$. Roberts / Vander Linden 2011, 5.

$61 \mathrm{Vgl}$. Hofmann 2009, 143-148.

62 Gotter 2001, 280. 
einer Objektbiographie ad absurdum führen, zumindest sofern der/die Protagonist/-in nicht nur für heute als ,bedeutend' gelten soll und/oder nur eine fiktionale Erzählung angestrebt wird. Zudem zeigt schon das Problem des Schiffs von Theseus ${ }^{63}$, dass mitunter zwar die materielle Identität eines Dinges noch bestimmt werden kann, damit über die Identität des Objektes ${ }^{64}$ aber noch nicht viel ausgesagt sein muss. In Anlehnung an Jürgen Straub und Joachim Renn könnte man wie bei Menschen auch bei Dingen von transitorischen Identitäten sprechen ${ }^{65}$, die im Zuge von Mensch-Ding-Relationen entstehen, verfestigt, in Frage gestellt, transformiert, verworfen werden oder auch verloren gehen können. Es sind übrigens gerade diese Veränderungen von Objektidentitäten, durch Gabentausch, Erbe, Veräußerungen, rites de passages, Performanzen, zentrale Ereignisse etc., die den Stoff für Objektbiographien bieten ${ }^{66}$. Die vielfach geäußerte Annahme, dass sich Bedeutungen und Geschichte bei Dingen stets akkumulieren, muss jedoch in Frage gestellt werden, denn Dinge können grundsätzlich auch ohne Wissenstransfer in ein anderes Wissenssystem gelangen, womit sie jedoch immer noch nicht ohne Vergangenheit wären ${ }^{67}$.

\section{DINGGESCHICHTEN: EIN ÜBERBLICK}

Ein Ding kann in den unterschiedlichsten Kontexten vorkommen, verschieden genutzt werden und differierende Bedeutungen zugeschrieben bekommen. Die Dauer seiner ,Lebensgeschichte‘ hängt vom Material, seiner Bearbeitung, aber auch von seiner Herstellung, dem Gebrauch, seinen Bedeutungs- und Wertzuschreibungen und Zugehörigkeiten zu Ding-Ensembles ab. Nicht nur welche Geschichten gelebt werden, sondern auch welche Geschichten erzählt werden, ändert sich. Geschichten mit Hilfe von Dingen bzw. Objekten zu erzählen, ist eine menschliche Kulturtechnik, die allerdings jenseits von Museen ${ }^{68}$ und der Wissenschaft in unserer modernen Konsumgesellschaft kaum mehr praktiziert wird.

63 Siehe u. a. Brown 2005. - Rosenberg 2006, 64-77.

64 Hoskins 2006, 74-75. - Cornelius Holtorf $(2002,55)$ hat hierfür den meines Erachtens missverständlichen Begriff „material identities“ gewählt.

$65 \mathrm{Vgl}$. Renn/Straub 2002.

66 Vgl. Gosden/Marshall 1999.

67 Siehe u. a. Eckardt/Williams 2003.

68 Vgl. MacGregor 2011. 


\subsection{ARCHÄOLOGISCHE UND ETHNOLOGISCHE DINGGESCHICHTEN}

Die Feststellungen, dass Objekte selbst Geschichten besitzen oder zugeschrieben bekommen, sind ebenfalls nicht neu, doch erst in den letzten Jahrzehnten hat dies in der Archäologie und Ethnologie zu den verschiedensten Ansätzen, Ding- und vor allem Objektgeschichten zu schreiben, geführt. Hier kann allerdings nur eine Auswahl und diese auch nur rudimentär vorgestellt werden.

Beginnen möchte ich als Kontrast zu den neueren Ansätzen mit dem Konzept des life cycle bzw. use-life des historical approach der new archaeolog ${ }^{69}$. Generalisierte Lebenszyklen und Prozesse von passiven Dingen werden hier vor allem anhand morphologischer und funktionaler Charakteristika beschrieben, um Technologie und Wirtschaft erfassen bzw. den archäologischen Befund in seiner Entstehung erklären zu können ${ }^{70}$. Flussdiagramme nutzend, unterschied Michael Brian Schiffer für Artefakte die ,Lebensphasen' Beschaffung, Herstellung, Nutzung, Recycling und/ oder Deponierung bzw. Beseitigung. Nach Übertritt in den archäologischen Kontext, theoretisch zu allen Lebensphasen denkbar, unterläge das Artefakt dann bis zu seiner Entdeckung nicht mehr kulturellen, sondern natürlichen Transformationen ${ }^{71}$.

Kopytoff ${ }^{72}$ interessierte sich in seinem objektbiographischen Ansatz indes vor allem für den Konsum und die Frage der Singularisierung und Kommodifizierung von Einzelobjekten und zwar vor dem Hintergrund idealer und potentieller Biographien. In emischer Perspektive wird so das social life von Dingen und Objekten einer Kultur untersucht ${ }^{73}$. Nicht mehr Funktion und Morphologie, sondern Änderung in der Bedeutung und in den Objektidentitäten von sich mitunter materiell nicht verändernden Dingen werden thematisiert.

In der Archäologie aufgegriffen - mit oder ohne Bezug auf Kopytoff wurden vor allem die Beschreibung realisierter Objektbiographien und die Frage nach der Veränderung der Bedeutung von Objekten durch soziale Interaktion. Neben Häusern ${ }^{74}$, Gräbern und Landschaften ${ }^{75}$ wurden vor allem Archaika bzw. Erbstücke, Spolien und Exotika oder umgearbeitete

69 Schiffer 1972; siehe ferner Dannehl 2009.

70 Tringham 1994, 175. - Gosden/Marshall 1999, 169.

71 Schiffer 1972.

72 Kopytoff 1986.

73 Vgl. Fontijn 2013.

74 z. B. Tringham 1994, 175. - Tringham 1995. - Gerritsen 1999.

75 Fontijn 2002. 
Dinge untersucht. Die Frage nach Kommodifizierung und Singularisierung spielte dabei jedoch weniger eine Rolle.

Cornelius Holtorf setzte sich in einem radikal-konstruktivistischen Ansatz dabei schon früh auch mit heutigen Wert- und Bedeutungszuschreibungen sowie der archäologischen Praxis und ihren Auswirkungen auf die von uns verfassten Geschichten auseinander ${ }^{76}$. Ihm dienten neben Grabmonumenten z. B. eine marginale Keramikscherbe als Protagonist einer Erzählung.

Die Anthropologin Janet Hoskins thematisierte mit dem Konzept der biographischen Objekte die Verflechtungen von Personen und Dinggeschichten im Rahmen von Selbst-Definitionen und persönlichen Erzählungen ${ }^{77}$. Objekte dienen hier als „Katalysatoren für biographische Berichte und Rekonstruktionen von Biographien ${ }^{478}$, da sie Erinnerungen evozieren.

Der Archäologe Jody Joy kombinierte unter dem Stichwort „relationale Biographien" verschiedene Konzepte, u. a. auch den der chaîne opératoire und des use-lifes, mit biographischen Ansätzen ${ }^{79}$. Dies diente ihm neben der Lösung des Problems fehlender Informationen auch dazu, Kausalität und Linearität zu relativieren. Ergänzend wird hier zudem auf die Relevanz von Performanz hingewiesen.

Hans-Peter Hahn und Hadas Weiss ${ }^{80}$ waren bis vor kurzem eine der ersten, die sich neben Matthias Jung ${ }^{81}$ kritisch mit den Nebenwirkungen bzw. Missverständnissen der sich immer mehr verselbstständigenden Metapher der Objektbiographie auseinandergesetzt haben. Das Konzept der travelling objects aufgreifend, plädieren sie dafür, anstelle von Biographie lieber von itineraries zu sprechen. Sie wollen unter diesem Begriff die nicht linear verlaufende Mobilität, die Bedeutungsveränderungen und Transformation von Objekten in Raum und Zeit beschreiben und dabei sowohl Zeiten der Reise als auch des Sesshaft-Seins beleuchten.

Doch welche Darstellungsformen und Erklärungsansprüche haben die verschiedenen Ansätze, Objekt- bzw. Dinggeschichten zu schreiben (Abb. 4)? Die new archaeology hat stark argumentierende Dinggeschichten verfasst, die nur grob zeitlich fixiert werden, um Langzeitentwicklungen nachzuvollziehen. Nomothetisch abstrahierend wurden vor allem wiederkehrende Zyklen und Prozesse thematisiert.

76 Holtorf 2000-2008. - Holtorf 2002.

77 Hoskins 1998; siehe auch Habermas 1996.

78 Hennig 2014, 234.

79 Joy 2009.

$80 \mathrm{Hahn} /$ Weiss 2013.

81 Jung 2012. 
use life, Taphonomie

(new archaeology) relational biographies

(Joy)

Itinerarien

(Hahn \& Hadas) object biography

(Kopytoff)

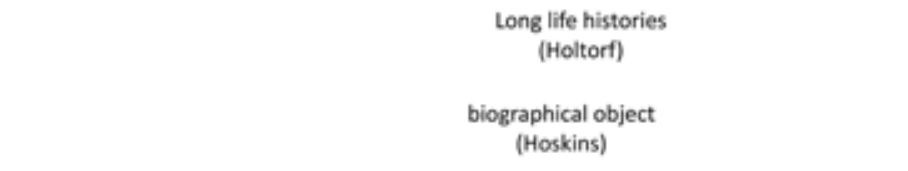

Argumentation

Ōberzeugung

generalisierend

kausale Zusammenhănge

herstellend

nicht zeitlich fixiert

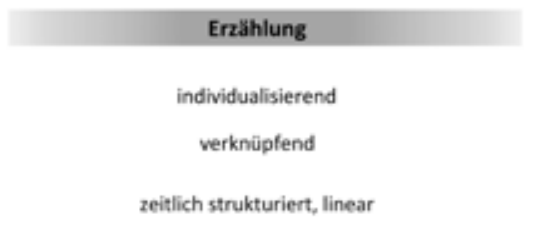

4 Archäologische und ethnologische Ding- und Objektgeschichten und ihre Darstellungsformen

Kopytoff widmete sich zwar insbesondere dem individualisierten Objekt, statt der Verfassung einer Objektbiographie geht es ihm aber darum, Prozesse der Kommodifizierung und Singularisierung zu verstehen. Daher würde ich seine bzw. eng nach seinem Konzept geschriebene Geschichten zwar als faktuale Erzählungen einstufen, aber noch mit einer Idee der Verallgemeinerung im Hinterkopf. Sowohl Holtorf als auch Hoskins schreiben bzw. geben Erzählungen wieder, in denen konkrete Objekte in spezifischen Mensch-Ding-Konstellationen Bedeutungen erhalten und Objektidentitäten sich verändert haben. Bei ihren Geschichten handelt es sich um MenschObjekt-Erzählungen. Joy und seine relationalen Biographien sind aus etischer Perspektive erfolgende, beschreibende Rekontextualisierungen. Dies trifft m. E. noch mehr für die Itinerarien von Hahn und Hadas zu. Linearität, Verallgemeinerung und Kausalitäten werden hier noch kritischer betrachtet und es wird nicht mehr vom Akkumulieren von Geschichten gesprochen.

\subsection{WILHELM SCHAPPS LEBENSGESCHICHTEN UND WOZUDINGE} Als Anregung für weitere mögliche Dinggeschichten, möchte ich im Folgenden Schapp und seine Geschichtenphilosophie vorstellen ${ }^{82}$. Wilhelm

82 Siehe auch Hofmann im Druck. 
Albert Johann Schapp (1884-1965) studierte bei Wilhelm Dilthey, Georg Simmel und Edmund Husserl. Nach seiner Promotion im Jahre 1909 über die „Phänomenologie der Wahrnehmung“ arbeitete er aber als Jurist. Erst nach dem Zweiten Weltkrieg widmete er sich als Privatmann wieder philosophischen Fragen und publizierte in den Jahren 1953 und 1959 die hier relevanten Werke „In Geschichten verstrickt. Zum Sein von Mensch und Ding“ und „Philosophie der Geschichten“ ${ }^{83}$.

Im Titel beider Werke fällt der Plural von Geschichten ins Auge. Schapp interessierten nicht die Fragen der klassischen Geschichtsphilosophie nach Herkunft und Zukunft unserer Zivilisation, sondern, was die Einheit unserer selbst in der Einheit unserer Lebenswelt ausmacht. Seine Antwort: es sind die Eigen-, Fremd- und Wir-Geschichten, in die wir alle - individuell wie kollektiv - „verstrickt ${ }^{\text {“84 }}$ bzw. „mitverstrickt“ sind, und die in ihrer Pluralität erzählt werden müssen, um nicht auf uns und unsere Freiheit zu verzichten ${ }^{85}$. Schapps Geschichtenphänomenologie ist mit der Daseinsanalytik Martin Heideggers verwandt und in ihren sprachanalytischen Teilen weist sie Nähe zu Ludwig Wittgenstein auf. Auf Dramatisierungen existentieller Befindlichkeiten und zivilisationskritische Zuspitzungen verzichtete Schapp jedoch ${ }^{86}$.

Schapp geht aus heutiger Sicht verwirrend unbefangen an Geschichten heran ${ }^{87}$. Ohne die Rolle des Erzählers zu problematisieren, beginnt er so z. B. sein erstes geschichtenphilosophisches Buch mit dem Satz „Wir Menschen sind immer in Geschichten verstrickt ${ }^{\text {"88 }}$. Geschichten sind bei ihm nichts Konstruiertes, d. h. nichts Zugeschriebes oder Erdachtes, sondern etwas in der Lebenswelt vorgefundenes, es sind auftauchende

83 Schapp 1953. - Schapp 1981. Für biographische Informationen siehe Lübbe 1993. - Haas 2002, 17-19.

84 Textile Metapher, um Beziehungen zu beschreiben, die Lebewesen und ihre Um- und Mitwelt verbindet, ohne immer zwischen aktiv und passiv Beteiligten unterscheiden zu müssen, wobei man sich diesen jedoch schwerlich entziehen kann. Im Gegensatz zum umgangssprachlichen Gebrauch sind diese mit Schapp aber nicht als negativ zu betrachten, denn die Freiheit wird nicht als bedroht angesehen, da man auch wählen kann, welchen Geweben man wie angehört, und welchen Bedeutungen man ihnen als Geschichten beimisst.

85 Marquard 2004, 50.

86 Lübbe 1993.

87 Haas 2002, 23.

88 Schapp 1953, 1. 
Zusammenhänge ${ }^{89}$. Als „Handlungs-Widerfahrnis-Gemische ${ }^{(900}$ sind sie weder naturgesetzliche Abläufe noch ausschließlich geplante Handlungen, sondern es sind die Kontingenzen, die Zufälle, die Geschichten ausmachen. Jeder Mensch ist dabei sein Lebenslauf, ein Ensemble aus Geschichten. Wichtig ist, dass man nicht nur eine Geschichte hat, sondern mehrere. Diese Pluralität bedarf es, um frei sein zu können. Geschichten müssen gelebt bzw. erzählt werden, um sie fortzusetzen. Wer darauf verzichtet, verzichtet auf seine Geschichte und damit sich selbst: nararre necesse est ${ }^{91}$.

Geschichten dienen ihm ferner zur Vermeidung des Substanz-AkzidenzSchemas ${ }^{92}$. So ist der Dackel Waldi, nicht der mit der kalten Schnauze und dem rosa Halsband, sondern derjenige, welcher Tante Rosalinde bei einem Picknick am Fluss gebissen hat, die dabei aus Schreck die Teetasse fallen ließ, die daraufhin einen Sprung hatte ${ }^{93}$. Damit sind zugleich die nach Schapp üblicherweise in Geschichten Vorkommenden genannt: Menschen - hier Tante Rosalinde -, Tiere - der Dackel Waldi -, Wozudinge - die bei ihm immer wieder als Beispiel herangezogene Tasse und die Außenwelt - der Fluss. Nur die Gebilde der Außenwelt, wie der Fluss, kommen zwar in Geschichten vor, sind aber nicht selbst in ihnen verstrickt, zumindest sofern sie nicht als Personen oder Lebewesen imaginiert werden ${ }^{94}$. Die Verbindung zwischen den Geschichten und der Außenwelt stellen die Wozudinge her, ,die von Menschen geschaffenen Dinge, wie Tische, Stühle, Tassen, Häuser, Paläste, die Werke der Menschen im Auge ${ }^{\text {“95 }}$. Jedes Wozuding hat zumindest eine Geschichte, da es von einem individuellen Menschen in einem Sinnzusammenhang zu irgendeinem Zweck geplant wurde ${ }^{96}$. Durch den Sprung in unserer

89 Eichler 2010, 108-109.

90 Marquard 2004, 50.

91 Marquard 2004, 45. 49-50.

92 Marquard 2004, 49-50. Mit dem von Aristoteles in die Philosophie eingeführten Substanz-Akzidenz-Schema wird zwischen dem Essentiellen und dem nicht wesentlichen, veränderbaren Anhängenden unterschieden. Im folgenden Satz wären der Dackel die Substanz, seine kalte Schnauze und das rosa Halsband die Akzidenzien.

93 Die hier in einem Satz erzählte Kurzgeschichte stammt von mir, entstand aber in Anlehnung an Wilhelm Schapps Geschichten, angeregt durch die Lektüre des eben zitierten Artikels zur Philosophie der Geschichten und der Zukunft des Erzählens von Odo Marquard.

94 Haas 2002, 24.

95 Schapp 1953, 3.

96 Schapp 1953, 2-3. 
Tasse taucht bereits eine weitere Geschichte auf, nämlich dass sie einmal nicht tassengemäß behandelt wurde und so nun einen Zweck möglicherweise nicht mehr erfüllen kann ${ }^{97}$. Auch ohne Erzählung lebt das oben wiedergegebene Geschehen so fort, allerdings sehr unkonkret in seinen Verstrickungen.

Wozudinge tauchen nach Schapp niemals nur punktuell gegenwärtig, sondern stets „mit einer Vergangenheit, mit einer Geschichte, mit einem Alter ${ }^{\text {"98 }}$ auf. Letzteres ist zudem eine der relationalen Bestimmtheiten von Wozudingen. Andere sind z. B. das Auswas und seine Größ $\beta e^{99}$. Diese sind nicht unabhängig existierend, sondern immer an das Wahrnehmungshandeln des Menschen geknüpft. Sie bieten weitere Möglichkeiten - auch für die Archäologie -, Geschichten zu erzählen. Doch bevor ich hier kurz ein paar mögliche Perspektiven aufzeigen will, sei hier noch ein wichtiger Aspekt hervorgehoben. Nach Schapp ist jedes Wozuding ein Individuum. Das vermeintlich Allgemeine, die Gattung findet man nirgends vor, es „tauchen nur Einzeldinge auf in einem festen Verbande“ aus vielfältigen Zusammenhängen ${ }^{100}$. Auch die Suche nach einem ersten Wozuding verliert sich somit „,irgendwo im Horizont ${ }^{\text {“101. }}$.

Eine zentrale Bestimmtheit des Wozudinges ist das Alter. Hier nicht als seine absolute oder relative Datierung verstanden, sondern als Eigenschaft, dass ein Wozuding mehr oder minder auffällig färbt. Ausdrücke wie veraltet, neu, gebraucht, verwahrlost sind relationale Bestimmtheiten von Wozudingen ${ }^{102}$, die an den Menschen und ihr Wahrnehmungshandeln sowie an die sie umgebenden Dinge gebunden sind. Interessant wäre es z.B. hier auf die Methode der Biographie von Sachgüter-Ausstattungen von Edith Fél und Jan Hofer ${ }^{103}$ zurückzugreifen, um festzustellen, wann ein Gegenstand als veraltet bzw. abgenutzt und somit vielleicht nicht mehr für ein Ding-Ensemble als geeignet gilt. In der Prähistorischen Archäologie würden sich hierfür zwar nur Glücksfunde wie Pompeji oder intentional deponierte Objekt-Sammlungen anbieten - und alternative Interpretationen müssten stets im Hinterkopf behalten werden - dennoch könnte die Frage z. B. bei einer vergleichenden Analyse von Weihegaben

97 Haas 2002, 25.

98 Schapp 1953, 17.

99 Schapp 1953, 11-82.

100 Schapp 1953, 58-59.

101 Schapp 1953, 28.

102 Schapp 1953, 13.

$103 \mathrm{Fel} /$ Hofer 1974. 
interessant sein. Ferner könnte man für Grabbeigaben fragen, ob die Wozudinge, die von als biographische Objekte angesprochen werden, nicht möglichst Gebrauchsspuren aufweisen sollten.

Ganz bewusst spricht Schapp bei einer anderen zentralen Bestimmtheit des Wozudinges vom Auswas und nicht von seinem Stoff oder seiner Substanz, denn letztere seien nicht etwas Selbstständiges, sondern würden erst während der Herstellung eines Wozudinges auftauchen ${ }^{104}$. Das Auswas des Wozudings verweist so unter anderem auch auf Pläne, Entschlüsse, Handlungen, Austauschbeziehungen, Vereinbarungen und Konventionen, den technischen Kenntnisstand der Produzent/-innen und ihre Lernprozesse. Das soziale und kulturelle Netzwerk der chaîne opératoire nach Pierre Lemonnier ${ }^{105}$ könnte man demnach versuchen, in Geschichten zu erfassen. Aber auch die Geschichte, warum man z.B. zu bestimmten Zeiten noch Bronze anstatt Eisen für rituelle Objekte gewählt hat, kann so am im Fundkontext auftauchenden Objekt geschrieben werden ${ }^{106}$. Generell könnte man Auswas-Geschichten von Wozudingen und ihre Wertsetzungen verfassen und statt der wenigen bisher mehr oder minder linear geschriebenen sogenannten Kulturgeschichten von Substanzen ${ }^{107}$, so noch stärker individuelle Verstrickungen und Besonderheiten herausarbeiten.

Eine weitere Wozudingbestimmtheit ist die Größ ${ }^{108}$. Jedes Wozuding hat seine bestimmte, ihm eigene Größe. Im Einzelfall könne diese von der ihm normalen Größe in einer gewissen Toleranz nach oben und unten abweichen, darüber hinaus verliere oder ändere sie jedoch den Charakter des Wozudings. Die Eigenschaft Groß oder Klein ist demnach beim Wozuding kein diesem immanenter Wert, sondern ergibt sich erst aus dem Zusammenhang, in den das Wozuding gestellt ist. In der Archäologie werden derartige Abweichungen von Wozudingen häufig unter den Begriffen Prestigegut und Miniaturen behandelt ${ }^{109}$. Aufgrund des externen Blicks auf fremde Kulturzusammenhänge erweist sich die Bestimmung der ,richtigen' Größe von Wozudingen in der Archäologie allerdings recht

104 Schapp 1953, 20-21. 31.

105 Lemonnier 1992.

106 Siehe Fontijn 2002, 28.

107 Exemplarisch seien hier eine Kulturgeschichte des Kaffees und Zucker genannt: Heise 2005. - Mintz 2007. Für ein Plädoyer zur stärkeren Berücksichtigung von Stoffen, siehe Hahn/Soentgen 2011.

108 Schapp 1953, 34-35.

109 Siehe exemplarisch Guggisberg 2009. - Notroff 2011. 
schwierig. So ist z. B. für Lanzetten, eine „Leitform“ der Nordischen Jüngeren Bronzezeit, trotz zahlreicher Analysen nicht eindeutig feststellbar, ob es sich um Miniaturen oder in ihrer Größe funktionale Artefakte handelt; vielmehr scheinen hier Form und Größe als Bestimmtheiten für eine zudem eher zu hinterfragende archäologische Fundkategorie nicht ausreichend, um das vielfältige Verstricktsein der einzelnen Wozudinge in Geschichten erfassen zu können ${ }^{110}$.

Versucht man die mit Schapps Ansatz überwiegend für die Archäologie erst noch zu schreibenden Wozuding-Geschichten einzuordnen, dann handelt es sich nicht um Biographien, sondern um Geschichten, die Zusammenhänge via Handlungen betonen. Der Zweck und der Gebrauch der Wozudinge, die jedoch auch Bedeutungen und Wertzuschreibungen beleuchten können, sind für ihn zentral. Voraussetzung für das Erzählen von Wozuding-Geschichten ist das Mitverstricktsein und für die Prähistorische Archäologie letztlich die konkrete Materialisierung von Geschichten im archäologischen (Be)Fund. Auch wenn Schapp selber vermutlich formulieren würde, dass er nur auftauchende Geschichten wiedergibt, handelt es sich bei diesen in der Darstellungsform dann doch eher um Erzählungen und weniger um Beschreibungen.

Schapps Geschichtenphilosophie taugt sicherlich nicht als Geschichtstheorie, schon allein weil er nicht zwischen Geschehen und Erzählen unterscheidet, eine Außenperspektive auf Fremdgeschichten ablehnt und die geschichtswissenschaftliche Forderungen nach Konsistenz, Widerspruchslosigkeit und Einklang mit den verfügbaren Quellen für ihn nicht zentral sind ${ }^{111}$. Auch birgt der universale Anspruch seiner narrativen Phänomenologie einige Fallstricke ${ }^{112}$. Für einige Vertreter/-innen der material culture studies ist vermutlich jedoch vor allem die für Wozudinge definierende Herstellung durch einen Menschen und der damit stets mitgedachte Primärzweck ein Dorn im Auge, versuchen sie doch verstärkt auf Materialität, Affordanz und Bedeutung von Dingen im allgemeinen, unabhängig von der Herstellungsintention, abzuheben ${ }^{113}$. Dennoch ist Schapps Geschichtenphilosophie m. E. sehr anregend. So kann sie zur Selbstreflexion dienen, wenn wir unsere eigenen pluralen Verstrickungen und die unserer Forschungsobjekte im Heute beleuchten. Ferner werden durch ihren Fokus auf Einzeldinge generalisierende Interpretationen

110 Hofmann 2004. - Hofmann/Schreiber 2011.

111 Siehe Scholtz 2004.

112 Siehe u. a. Eichler 2010.

113 z. B. Ingold 2008. - Schreiber 2013. - Keßeler im Druck. 
erschwert. Zudem können materialisierte Lebensgeschichten mit Dingen geschrieben werden, die Menschen, aber auch Tiere thematisieren, ohne sie in ihrer Handlungsfähigkeit mit Wozudingen gleichzusetzen. Dabei kommt die Geschichtenphilosophie, im Gegensatz zu aktuellen MenschDing-Verflechtungsgeschichten ${ }^{114}$, ohne evolutionistischen Impetus aus. Vielmehr wird durch die Betonung des hohen Werts der Geschichtenpluralität zum narrativen Interpretieren auch jenseits der üblichen, sich oft verselbstständigenden Interpretationsmuster aufgerufen.

\section{VON HISTORY ZU ITSTORIES?}

Bedeutet dies jedoch zugleich den zwangsläufigen Weg von History zu $i t$-stories? Die Geschichte interessierte Schapp nicht und sähe er, wenn sie als alleingültige Metanarrative erzählt würde, als freiheitseinschränkend an. Auch Objektbiographien zu schreiben, lag ihm fern. Es sind die pluralen Lebensgeschichten und die Verstrickungen von Wozudingen mit Menschen und Tieren sowie ihre Nahtstellen-Funktion zur Außenwelt ${ }^{115}$, die ihn mit dem Ziel interessieren, den Menschen und seine Lebenswelt besser zu verstehen. Ein emanzipatorischer Impetus, wie die hier gestellte Frage suggeriert, ist ihm dabei jedoch fremd. Je nach Verstrickungsgrad und Auftauchen dürfte es nach Schapp einfacher fallen, Mensch-Dingoder Ding-Mensch-Geschichten zu schreiben. Reine Dinggeschichten gibt es bei ihm zu recht jedoch nicht.

Ob nun die von Kopytoff gewählte Metapher „Objektbiographie“ passend bzw. gar erkenntnisbringend war bzw. ist, hängt sehr davon $a b$, welchen Zeitraum man betrachtet und ob man Kopytoff für die Nebenwirkungen, die Ausweitung seines Konzeptes und die nahezu beliebige Nutzung der Metapher verantwortlich machen will. Die Bezeichnung „Objektbiographie“ ist bei ihm jedenfalls kein „Nebelwerfer der Hilflosigkeit ${ }^{\star 1116}$, sondern eine für seine Forschungsziele sehr eingängige Bezeichnung. Festzuhalten ist ferner, dass unter Rekurs auf sein Konzept bzw. seine Metapher eine große Anzahl von z.T. sehr innovativen Forschungen entstanden ist. Nach George Lakoff und Mark Johnson und Andrew Goatly gibt es „metaphors we live by“ und „meaphors we

114 z. B. Chapman/Gaydarska 2009. - Hodder 2012.

115 Schapp 1953, 3.

116 Finke 2013, 47. 
die by ${ }^{\text {“117 }}$. Die Kulturwissenschaften wurden durch die ObjektbiographieMetapher zunächst sehr belebt, allerdings zeigte sich bei eingehender Betrachtung, dass die Metapher doch z. T. recht problematisch ist und nicht nur wünschenswerte Pfade erschließt (siehe Beitrag Hahn).

Für die Prähistorische Archäologie war m. E. sehr vorteilhaft, dass man stärker über die Veränderungen von Objekt-Bedeutungen und -Identitäten reflektierte und nun z. B. gezielter nach Konsum und Aneignung von Objekten fragen konnte, aber auch Rezeptionen und heutige archäologische Praxis in den Blick gerieten. Schwierig wird es jedoch, wenn wir verzweifelt ganze Biographien von der Geburt bis zum Tode schreiben wollen. Hier sind unsere Informationsquellen äußerst schlecht und wir müssen immer wieder auf Allgemeinplätze zurückgreifen, die eigentlich nicht zentraler Teil von Erzählungen sein sollten. Zudem werden wir als Erzählende im Sinne des „story bias“ ${ }^{118}$ immer wieder der Versuchung ausgesetzt, Kausalbezüge herzustellen, die so nicht überliefert und aufgrund der Nutzung von dominanten Erzählmustern und plausibel erscheinenden Erklärungsansätzen eben nicht zur Historisierung, sondern allenfalls zur Simplifizierung beitragen. Insbesondere die bei der Analyse von Dingen so wichtigen Brüche werden von Ding-Geschichten meist - wenn sie nicht als Beginn oder Ende konzeptualisiert sind - letztlich aufgrund der notwendigen Kontinuität der Handlungsträger/-innen relativiert. Die Bezeichnung „Objektbiographie“ droht derzeit daher immer wieder von einer "we live by" zu einer „we die by"-Metapher zu werden. Ähnlich wie das Bild der, Gräber - Spiegel bzw. Zerrspiegel des Lebens ${ }^{{ }^{\varsigma 119}}$ ist vermutlich auch diese für die Archäologie nur einer unter vielen ,Lebensabschnittsgefährten'. Jenseits eines programmatischen catch-allBegriffes geht es m. E. inzwischen darum, differenziert zu schauen, welche Text- und Erzählformen auf welcher Grundlage und Zielsetzung eigentlich angestrebt und umgesetzt wurden bzw. werden.

Kommen wir noch einmal zur Frage „Von History zu itstories?“ zurück. Ein gewisser Trend von der großen Menschheitserzählung mit überwiegend weißen Protagonisten zur Pluralität von Geschichten u.a. mit Objekten als Hauptfiguren ist nicht von der Hand zu weisen, obwohl immer noch oder in der Archäologie gerade wieder neue Metanarrative geschrieben werden. Die stärkere Berücksichtigung von Dingen und Objekten, auch als „Wandler zwischen bzw. von Dichotomien“, ist aus Sicht

117 Lakoff/Johnson 1980. - Goatly 1996. - Goatly 1997.

118 Dobelli 2011, 53-56.

119 Hofmann 2013, 286. 
einer überwiegend mit diesen arbeitenden Prähistorikerin erfreulich. Wichtig ist mir dabei jedoch - wie übrigens in allen den hier vorgestellten Ansätzen auch zu erkennen - den Menschen als Handlungstragenden nicht zu verlieren. Da Menschen, Dinge und ihre Identitäten im Leben miteinander verstrickt sind, sollten sie auch in den geschichtswissenschaftlichen Erzählungen gemeinsam thematisiert werden.

\section{ABBILDUNGSNACHWEIS}

1-4 K. P. Hofmann.

\section{BIBLIOGRAPHIE}

Ankersmit 1983 Ankersmit, F. R.: Narrative logic. A semantic analysis of the historian's language. Den Haag 1983.

Appadurai 1986 Appadurai, A.: Introduction. Commodities and the politics of value. In: A. Appadurai (Hrsg.), The Social Life of Things. Commodities in Cultural Perspective. Cambridge 1986, 3-63.

Arendt 2011 Arendt, H.: Vita activa oder Vom tätigen Leben. München ${ }^{10} 2011$. Bachmann-Medick 2014 Bachmann-Medick, D.: From Hybridity to Translation. Reflections on Travelling Concepts. In: D. Bachmann-Medick (Hrsg.): The Trans/National Study of Culture. A Translational Perspective, Concepts for the study of culture 4. Berlin 2014, 119-136.

Barthes 1988 Barthes, R.: Einführung in die strukturale Analyse von Erzählungen. 1966. In: R. Barthes (Hrsg.): Das semiologische Abenteuer. Frankfurt a. M. 1988, 102-143.

Belliger/Krieger (Hrsg.) 2006 Belliger, A. / Krieger, D. J. (Hrsg.): ANThology. Ein einführendes Handbuch zur Akteur-Netzwerk-Theorie, ScienceStudies. Bielefeld 2006.

Bernbeck 2010 Bernbeck, R.: „La Jalousie“ und Archäologie. Plädoyer für subjektloses Erzählen, Ethnographisch-Archäologische Zeitschrift 51, 2010, 64-86.

Brown 2001 Brown, B.: Thing Theory, Critical Inquiry 28, 2001, 1-22.

Brown 2005 Brown, C. M.: Aquinas and the Ship of Theseus. Solving Puzzles about Material Objects. London / New York 2005. 
Bruner 1986 Bruner, E. M.: Ethnography as Narrative. In: V. W. Turner / E. M. Bruner (Hrsg.): The Anthropology of experience. Urbana 1986, 139-155.

Cancik-Kirschbaum/Wiedemann in Vorbereitung Cancik-Kirschbaum, E. / Wiedemann, F.: „Wer sind denn die Semiten?“. Namentliche Identifizierungen und narrative Identitäten in den Altertumswissenschaften (Manuskript in Vorbereitung).

Carr 1986 Carr, D.: Narrative and the Real World. An Argument for Continuity, History and Theory 25, 1986, 117-131.

Chapman 2000 Chapman, J.: Fragmentation in Archaeology. People, Places, and Broken Objects in the Prehistory of South Eastern Europe. London / New York 2000.

Chapman/Gaydarska 2009 Chapman, J. / Gaydarska, B.: The Fragmentation Premise in Archaeology. In: W. Tronzo (Hrsg.): The Fragment. An Incomplete History. Los Angeles 2009, 130-153.

Chatman 1990 Chatman, S. B.: Coming to Terms. The Rhetoric of Narrative in Fiction and Film. Ithaca 1990.

Dannehl 2009 Dannehl, K.: Object Biographies. From Production to Consumption. In: K. Harvey (Hrsg.): History and Material Culture. A Student's Guide to Approaching Alternative Sources. London 2009, 123-138.

Derrida 1984 Derrida, J.: Signéponge = Signsponge. New York 1984.

Dilthey 1927 Dilthey, W.: Der Aufbau der geschichtlichen Welt in den Geisteswissenschaften. Wilhelm Diltheys gesammelte Schriften 7. Leipzig/ Berlin 1927.

Dobelli 2011 Dobelli, R.: Die Kunst des klaren Denkens. 52 Denkfehler, die Sie besser anderen überlassen. München 2011.

Dobres/Robb 2000 Dobres, M.-A. / Robb, J. E. (Hrsg.): Agency in Archaeology. London / New York 2000.

Eckardt/Williams 2003 Eckardt, H. / Williams, H.: Objects without a Past? The use of Roman objects in early Anglo-Saxon graves. In: H. Williams (Hrsg.): Archaeologies of Remembrance. Death and Memory in Past Societies. New York 2003, 141-170.

Eggert 2006 Eggert, M. K. H.: Archäologie. Grundzüge einer Historischen Kulturwissenschaft. Tübingen 2006.

Eichler 2010 Eichler, K.-D.: Wilhelm Schapps narrative Ontologie. Eine Problematisierung seiner Geschichtenphilosophie. In: K. Joisten / N. Thiemer (Hrsg.): Das Denken Wilhelm Schapps. Perspektiven für unsere Zeit 21. Freiburg i. Br. 2010, 102-125.

Fel/Hofer 1974 Fel, E. / Hofer, T.: Geräte der Átányer Bauern, Kommission der Königlich Dänischen Akademie der Wissenschaften zur Erforschung der Geschichte der Ackerbaugeräte und der Feldstrukturen 2. Budapest 1974. 
Fetz 2009 Fetz, B.: Die vielen Leben der Biographie. Interdisziplinäre Aspekte einer Theorie der Biographie. In: B. Fetz / H. Schweiger (Hrsg.): Die Biographie, zur Grundlegung ihrer Theorie. Berlin / New York 2009, 3-66.

Finke 2013 Finke, P.: Misteln, Wälder und Frösche. Über Metaphern in der Wissenschaft, Metaphorik 04, 2013, 45-65. <http://www.metaphorik.de/sites/ www.metaphorik.de/files/journal-pdf/04_2003_finke.pdf $>(07.05 .2014)$

Fludernik 2010 Fludernik, M.: Erzähltheorie. Eine Einführung, Einführung Literaturwissenschaft. Darmstadt ${ }^{3} 2010$.

Fontijn 2002 Fontijn, D. R.: Sacrificial Landscapes. Cultural Biographies of Persons, Objects and ,Natural' Places in the Bronze Age of the Southern Netherlands, C. 2300-600 BC, Analecta praehistorica Leidensia 33/34. Leiden 2002.

Fontijn 2013 Fontijn, D.: Epilogue. Cultural biographies and itineraries of things. Second thoughts. In: H. P. Hahn / H. Weis (Hrsg.): Mobility, Meaning \& Transformation of Things. Shifting Contexts of Material Culture through Time and Space. Oxford 2013, 183-195.

Forster 1985 Forster, E. M.: Aspects of the novel. A Harvest/HBJ book. San Diego / New York / London ${ }^{3} 1985$.

Frers 2009 Frers, L.: Herausfordernde Materialitäten. Gegenstände, Methoden, Konzepte, Berichte zur deutschen Landeskunde 83, 2009, 177-191.

Geertz 1993 Geertz, C.: Die künstlichen Wilden. Der Anthropologe als Schriftsteller. Frankfurt a. M. 1993.

Gell 1998 Gell, A.: Art and Agency. An Anthropological Theory. Oxford 1998.

Gerritsen 1999 Gerritsen, F.: To build and to abandon. The cultural biography of late prehistoric houses and farmsteads in southern Netherlands, Archaeological Dialogues 6, 1999, 78-97.

Goatly 1996 Goatly, A.: Green grammar and grammatical metaphor, or language and the myth of power, or metaphors we die by, Journal of Pragmatics 25, 1996, 537-560

Goatly 1997 Goatly, A.: The language of metaphors. London / New York 1997.

Goetz 1993 Goetz, H.-W.: Proseminar Geschichte. Mittelalter. Stuttgart 1993.

Gosden 2005 Gosden, C.: What Do Objects Want?, Journal of Archaeological Method and Theory 12, 2005, 193-211.

Gosden/Marshall 1999 Gosden, C. / Marshall, Y.: The cultural biography of objects, World Archaeology 31, 1999, 169-178.

Gotter 2001 Gotter, U.: ,Akkulturation' als Methodenproblem der historischen Wissenschaften. In: S. Altekamp / M. Krumme / M. R. Hofter (Hrsg.): Posthumanistische Klassische Archäologie. Historizität und Wissenschaftlichkeit 
von Interessen und Methoden. Kolloquium Berlin 1999. München 2001, 255-280.

Greimas 1971 Greimas, A. J.: Strukturale Semantik. Methodologische Untersuchungen. Braunschweig 1971.

Guggisberg 2009 Guggisberg, M.: Größe als Gabe. Gedanken zum Format von „Prestigegütern“ in frühen Kulturen der Mittelmeerwelt und ihrer Randzone. In: B. Hildebrandt / C. Veit (Hrsg.): Der Wert der Dinge - Güter im Prestigediskurs. „Formen von Prestige in Kulturen des Altertums“ Graduiertenkolleg der DFG an der Ludwig-Maximilians-Universität München. München 2009, 103-141.

Haas 2002 Haas, S.: Kein Selbst ohne Geschichten. Wilhelm Schapps Geschichtenphilosophie und Paul Ricœurs Überlegungen zur narrativen Identität, Studien und Materialien zur Geschichte der Philosophie 61. Hildesheim / Zürich / New York 2002.

Habermas 1996 Habermas, T.: Geliebte Objekte. Symbole und Instrumente der Identitätsbildung, Perspektiven der Humanwissenschaften. Phänomenologisch-psychologische Forschungen 19. Berlin / New York 1996.

Hahn 2011 Hahn, H. P.: Konsumlogik und Eigensinn der Dinge. In: H. Drügh / C. Metz / B. Weyand (Hrsg.): Warenästhetik. Neue Perspektiven auf Konsum, Kultur und Kunst. Frankfurt a. M. 2011, 92-110.

Hahn 2013 Hahn, H. P.: Vom Eigensinn der Dinge, Bayerisches Jahrbuch für Volkskunde 2013, 13-22.

Hahn/Soentgen 2011 Hahn, H. P. / Soentgen, J.: Acknowledging Substances. Looking at the Hidden Side of the Material World, Philosophy and Technology 24, 2011, 19-33.

Hahn/Weiss 2013 Hahn, H. P. / Weiss, H.: Introduction. Biographies, travels and itineraries of things. In: H. P. Hahn / H. Weis (Hrsg.): Mobility, Meaning \& Transformation of Things. Shifting Contexts of Material Culture through Time and Space. Oxford 2013, 1-14.

Hampe 2007 Hampe, M.: Eine kleine Geschichte des Naturgesetzbegriffs. Frankfurt a. M. 2007.

Haraway 1995 Haraway, D.: Ein Manifest für Cyborgs. Feminismus im Streit mit den Technowissenschaften. In: D. Haraway (Hrsg.): Die Neuerfindung der Natur. Primaten, Cyborgs und Frauen. Frankfurt a. M. / New York 1995, 33-72.

Hartmann 1962 Hartmann, N.: Ethik. Berlin ${ }^{4} 1962$.

Heise 2005 Heise, U.: Kaffee und Kaffeehaus. Eine Bohne macht Kulturgeschichte. Köln 2005.

Hennig 2014 Hennig, N.: Objektbiographie. In: S. Samida / M. K. H. Eggert / H. P. Hahn (Hrsg.): Handbuch Materielle Kultur. Bedeutungen, Konzepte, Disziplinen. Stuttgart 2014, 234-237. 
Hodder 2012 Hodder, I.: Entangled. An archaeology of the relationships between humans and things. Malden 2012.

Hofmann 2004 Hofmann, K. P.: Lanzetten. Eine Leitform der Nordischen Bronzezeit. Fundanalyse im Spannungsfeld zwischen Theorie und Praxis. In: S. Hesse (Hrsg.): Spurensicherung. 25 Jahre Kreisarchäologie Rotenburg (Wümme), Archäologische Berichte des Landkreises Rotenburg (Wümme) 11. Oldenburg 2004, 105-222.

Hofmann 2009 Hofmann, K. P.: Grabbefunde zwischen sex und gender. In: U. Rambuscheck (Hrsg.): Zwischen Diskursanalyse und Isotopenforschung. Methoden der archäologischen Geschlechterforschung. Bericht der 3. Sitzung der AG Geschlechterforschung auf der 78. Tagung des Nordwestdeutschen Verbandes für Altertumsforschung e.V. in Schleswig 2007, Frauen, Forschung, Archäologie 8. Münster / New York / München / Berlin 2009, 133-161.

Hofmann 2013 Hofmann, K. P.: Gräber und Totenrituale. Zu aktuellen Theorien und Forschungsansätzen. In: M. K. H. Eggert / U. Veit (Hrsg.): Theorie in der Archäologie. Zur jüngeren Diskussion in Deutschland. Tübinger Archäologische Taschenbücher 10. Münster / New York / München / Berlin 2013, 269-298.

Hofmann 2014 Hofmann, K. P.: Auf der Suche nach der Jastorf-Fibel. Die ältereisenzeitlichen Plattenfibeln Norddeutschlands - eine Leitform? In: J. Brandt / B. Rauchfuß (Hrsg.): Das Jastorf-Konzept und die vorrömische Eisenzeit im nördlichen Mitteleuropa. Beiträge der Internationalen Tagung zum einhundertjährigen Jubiläum der Veröffentlichung der Ältesten Urnenfriedhöfe bei Uelzen und Lüneburg durch Gustav Schwantes 18. - 22.05.2011 in Bad Bevensen, Veröffentlichungen des Archäologischen Museums Hamburg 105. Hamburg 2014, 129-142.

Hofmann im Druck Hofmann, K. P.: Dinge als historische Quellen in Revision. Materialität, Spuren und Geschichten. In: Hofmann u. a. im Druck.

Hofmann u. a. im Druck Hofmann, K. P. / Meier, T. / Mölders, D. / Schreiber, S. (Hrsg.): Massendinghaltung in der Archäologie. Der material turn und die Ur- und Frühgeschichte (im Druck).

Hofmann/Schreiber 2011 Hofmann, K. P. / Schreiber, S.: Mit Lanzetten durch den practical turn. Zum Wechselspiel zwischen Mensch und Ding aus archäologischer Perspektive, Ethnographisch-Archäologische Zeitschrift 52, 2011, 163-187.

Holtorf 2000-2008 Holtorf, C.: Monumental Past. The Life-histories of Megalithic Monuments in Mecklenburg-Vorpommern (Germany). Electronic monograph, <http://hdl.handle.net/1807/245> (29.11.2012).

Holtorf 2002 Holtorf, C.: Notes on the Life History of a Pot Sherd, Journal of Material Culture 7, 2002, 49-71. 
Hoskins 1998 Hoskins, J.: Biographical Objects. How Things Tell the Stories of People's Lives. New York / London 1998.

Hoskins 2006 Hoskins, J.: Agency, Biography and Objects. In: C. Tilley / W. Keane / S. Küchler / M. Rowlands / P. Spyer (Hrsg.): Handbook of Material Culture. London 2006, 74-84.

Ingold 2008 Ingold, T.: When ANT meets SPIDER. Social theory for arthopods. In: C. Knappett / L. Malafouris (Hrsg.): Material Agency. Towards a Non-Anthropocentric Approach. New York 2008, 209-215.

Ivleva im Druck Ivleva, T.: A Totality of a Thing with Objects. Multifaceted British-made Brooches Abroad. In: Hofmann u. a. im Druck.

Jackman/Witmore 2002 Jackman, T. / Witmore, C. L.: Editorial. The narrative act and archaeology, Stanford Journal of Archaeology 1, 2002, <http://www. stanford.edu/dept/archaeology/journal/newdraft/editorial.html $>$.

Jaeger 2002 Jaeger, S.: Erzähltheorie und Geschichtswissenschaft. In: V. Nünning / A. Nünning (Hrsg.): Erzähltheorie transgenerisch, intermedial, interdisziplinär. Trier 2002, 237-263.

Johansson 1976 Johansson, S. R.: „Herstory“ as History. A New Field or Another Fad? In: B. A. Carroll (Hrsg.): Liberating women's history. Theoretical and critical essays. Urbana 1976, 400-430.

Joy 2009 Joy, J.: Reinvigorating object biography. Reproducing the drama of object lives, World Archaeology 41, 2009, 540-556.

Jung 2010 Jung, M.: Hofberichterstattung. Zur Wirkmächtigkeit des narrativen Ideals in der Hallstattforschung, Ethnographisch-Archäologische Zeitschrift 51, 2010, 151-172.

Jung 2012 Jung, M.: „Objektbiographie“ oder „Verwirklichung objektiver Möglichkeiten“? Zur Nutzung und Umnutzung eines Steinbeiles aus der Côte d'Ivoire. In: B. Ramminger / H. Lasch (Hrsg.): Hunde - Menschen Artefakte. Gedenkschrift für Gretel Gallay, Internationale Archäologie. Studia honoraria 32. Rahden/Westf. 2012, 375-383.

Keßeler in Vorbereitung Keßeler, A.: Der/Die/Das Gender der Dinge. In: M. Fotta / H. P. Hahn / P. W. Stockhammer (Hrsg.): Lost in Things. Questioning Functions and Meanings of the Material World. Internationale Konferenz am 28./29. November 2013 in Frankfurt a. M. (in Vorbereitung).

Kim 2002 Kim, T.: Vom Aktantenmodell zur Semiotik der Leidenschaften. Eine Studie zur narrativen Semiotik von Algirdas J. Greimas. Tübingen 2002.

Kistler 2010 Kistler, E.: Großkönigliches symbolon im Osten - exotisches Luxusgut im Westen. Zur Objektbiographie der achämenidischen Glasschale aus Ihringen. In: R. Rollinger (Hrsg.): Interkulturalität in der Alten Welt. Vorderasien, Hellas, Ägypten und die vielfältigen Ebenen des 
Kontakts, Philippika. Marburger altertumskundliche Abhandlungen 34 . Wiesbaden 2010, 63-96.

Kleemann u. a. 2007 Kleemann, F. / Krähnke, U. / Matuschek, I.: Interpretative Sozialforschung. Eine Einführung in die Praxis des Interpretierens. Wiesbaden 2007.

Klein/Martinez 2009 Klein, C. / Martinez, M. (Hrsg.): Wirklichkeitserzählungen. Felder, Formen und Funktionen nicht-literarischen Erzählens. Stuttgart 2009.

Knappett/Malafouris 2008 Knappett, C. / Malafouris, L. (Hrsg.): Material Agency. Towards a Non-Anthropocentric Approach. New York 2008.

Kneer u. a. 2008 Kneer, G. / Schroer, M. / Schüttpelz, E. (Hrsg.): Bruno Latours Kollektive. Kontroversen zur Entgrenzung des Sozialen. Frankfurt a. M. 2008.

Knigge 2011 Knigge, V.: Vom Zeugniswert der authentischen Substanz für die Gedenkstättenarbeit. In: A. Klausmeier / G. Schlusche (Hrsg.): Denkmalpflege für die Berliner Mauer. Die Konservierung eines unbequemen Bauwerks, Beiträge zur Geschichte von Mauer und Flucht. Berlin 2011, 65-71.

Kohl 1992 Kohl, K.-H.: Geordnete Erfahrung. Wissenschaftliche Darstellungsformen und literarischer Diskus in der Ethnologie. In: J. Matthes (Hrsg.): Zwischen den Kulturen? Die Sozialwissenschaften vor dem Problem des Kulturvergleichs, Soziale Welt. Sonderband 8. Göttingen 1992, 363-374.

Kohl 2003 Kohl, K.-H.: Die Macht der Dinge. Geschichte und Theorie sakraler Objekte. München 2003.

Kopytoff 1986 Kopytoff, I.: The Cultural Biography of Things. Commoditization as Process. In: A. Appadurai (Hrsg.): The Social Life of Things. Commodities in Cultural Perspective. Cambridge 1986, 64-91.

Koschorke 2012 Koschorke, A.: Wahrheit und Erfindung. Grundzüge einer Allgemeinen Erzähltheorie. Frankfurt a. M. ${ }^{2} 2012$.

Koselleck 1979 Koselleck, R.: Vergangene Zukunft. Zur Semantik geschichtlicher Zeiten. Frankfurt a. M. 1979.

Lakoff/Johnson 1980 Lakoff, G. / Johnson, M.: Metaphors we live by. Chicago 1980.

Latour 2005 Latour, B.: Reassembling the Social. An Introduction to ActorNetwork-Theory. Oxford / New York 2005.

Lemonnier 1992 Lemonnier, P.: Elements for an Anthropology of Technology, Anthropological Papers. Museum of Anthropology, University of Michigan 88. Ann Arbor 1992. 
Leskovar 2005 Leskovar J.: ArchäologInnengarn. Vom Nutzen erzählender und mehrfacher Deutung prähistorischer Evidenz. In: R. Karl - J. Leskovar (Hrsg.): Interpretierte Eisenzeiten. Fallstudien, Methoden, Theorie. Tagungsbeiträge der 1. Linzer Gespräche zur interpretativen Eisenzeitarchäologie, Studien zur Kulturgeschichte von Oberösterreich 18. Linz 2005, 131-145.

Lübbe 1993 Lübbe, H.: Schapp, Wilhelm Albert Johann. In: M. Tielke (Hrsg.): Biographisches Lexikon für Ostfriesland. Aurich 1993, 302-305.

Ludwig 2011 Ludwig, A.: Materielle Kultur. Version: 1.o, $<$ https://docupedia.de/ $\mathrm{zg} /$ Materielle_Kultur?oldid=78675 $>$ (10.04.2012).

Lupton 2006 Lupton, C.: The Knowing Book. Authors, It-Narratives, and Objectification in the Eighteenth Century, NOVEL. A Forum on Fiction 39, 2006, 402-420.

MacGregor 2011 MacGregor, N.: A history of the world in 100 objects. London 2011.

MacIntyre 1995 MacIntyre, A. C.: Der Verlust der Tugend. Zur moralischen Krise der Gegenwart. Frankfurt a. M. ${ }^{51995 .}$

Marquard 2004 Marquard, O.: Die Philosophie der Geschichten und die Zukunft des Erzählens. In: K.-H. Lembeck (Hrsg.): Geschichte und Geschichten. Studien zur Geschichtenphänomenologie Wilhelm Schapps 7. Würzburg 2004, 45-56.

Meuter 1995 Meuter, N.: Narrative Identität. Das Problem der personalen Identität im Anschluß an Ernst Tugendhat, Niklas Luhmann und Paul Ricoeur. Stuttgart 1995.

Mink 1970 Mink, L. O.: History and Fiction as Modes of Comprehension, New Literary History 1, 1970, 541-558.

Mintz 2007 Mintz, S. W.: Die süße Macht. Kulturgeschichte des Zuckers. Frankfurt a. M. ${ }^{2} 2007$.

Morin 1969 Morin, V.: L'objet biographique, Communications 13, 1969, 131-139.

Müller/Rüsen 1997 Müller, K. E. / Rüsen, J. (Hrsg.): Historische Sinnbildung. Problemstellungen, Zeitkonzepte, Wahrnehmungshorizonte, Darstellungsstrategien. Reinbek bei Hamburg 1997.

Notroff 2011 Notroff, J.: Vom Sinn der kleinen Dinge. Überlegungen zur Ansprache und Deutung von Miniaturgefäßen am Beispiel der Funde von Tall Ḥujayrāt al-Ghuzlān, Jordanien, Zeitschrift für Orient-Archäologie 4, 2011, 246-260.

Nünning 2012 Nünning, A.: Narrativist Approaches and Narratological Concepts for the Study of Culture. In: B. Neumann / A. Nünning (Hrsg.): Travelling concepts for the study of culture 2, Concepts for the study of culture 2 . Berlin/Boston 2012, 145-183. 
Olsen 2010 Olsen, B.: In Defense of Things. Archaeology and the Ontology of Objects. Archaeology in Societiy Series. Lanbam / Boulder / New York / Toronto / Oxford 2010.

Olsen u. a. 2012 Olsen, B. / Shanks, M. / Webmoor, T. / Witmore, C. L.: Archaeology. The discipline of things. Berkeley / Los Angeles / London 2012.

Paul/Schossig 1986 Paul, G. / Schossig, B. (Hrsg.): Die Andere Geschichte. Geschichte von unten, Spurensicherung, ökologische Geschichte, Geschichtswerkstätten. Köln 1986.

Pearce 1993 Pearce, S. M.: Museums, Objects, and Collections. A cultural study. Washington ${ }^{2} 1993$.

Pétursdóttir 2012 Pétursdóttir, P.: Concrete matters. Ruins of modernity and the things called heritage, Journal of Social Archaeology 13, 1, 2012, 31-53.

Pluciennik 1999 Pluciennik, M.: Archaeological Narratives and Other Ways of Telling, Current Anthropology 40, 1999, 653-678.

Pollock u. a. 2014 Pollock, S. / Bernbeck, R. / Jauß, C. / Greger, J. / von Rüden, C. / Schreiber, S.: A Discussion with Ian Hodder on his book Entangled. An Archaeology of the Relationships between Humans and Things. Berlin, 13. December 2013, Forum Kritische Archäologie 3, 2014, 151-161. <http://www. kritischearchaeologie.de/repositorium/fka/2014_11_hodder.pdf $>$ (26.11.2014).

Renn/Straub 2002 Renn, J. / Straub, J.: Transitorische Identität. Der Prozesscharakter moderner personaler Selbstverhältnisse. In: J. Straub / J. Renn (Hrsg.): Transitorische Identität. Der Prozesscharakter des modernen Selbst. Frankfurt a. M. / New York 2002, 10-31.

Ricœur 1986 Ricœur, P.: Zufall und Vernunft in der Geschichte. Tübingen 1986.

Ricœur 1996 Ricœur, P.: Das Selbst als ein Anderer. Übergänge. Texte und Studien zu Handlung, Sprache und Lebenswelt 26. München 1996.

Ricœur 2007a Ricœur, P.: Zeit und Erzählung. Band I: Zeit und historische Erzählung, Übergänge 18,I. München ${ }^{2} 2007$.

Ricœur 2007b Ricœur, P.: Zeit und Erzählung. Band II: Zeit und literarische Erzählung, Übergänge 18,II. München ${ }^{2} 2007$.

Rieckhoff im Druck Rieckhoff, S.: Ist das Archäologie oder kann das weg? Zur Konvergenz von Archäologie und Kunst. In: Hofmann u. a. im Druck.

Rieckhoff u. a. 2010 Rieckhoff, S. / Veit, U. / Wolfram, S.: Der Archäologe als Erzähler, Ethnographisch-Archäologische Zeitschrift 51, 2010, 7-9.

Roberts / Vander Linden 2011 Roberts, B. W. / Vander Linden, M.: Investigating Archaeological Cultures. Material Culture, Variability and Transmission. In: B. W. Roberts / M. Vander Linden (Hrsg.): Investigating archaeological cultures. Material culture, variability, and transmission. New York 2011, 1-21. 
Rosenberg 2006 Rosenberg, J. F.: Philosophieren. Ein Handbuch für Anfänger. Frankfurt a. M. ${ }^{5} 2006$.

Ruffing 2009 Ruffing, R.: Bruno Latour. Paderborn 2009.

Rübel 2009 Rübel, D.: Dinge werden Kunst - Dinge machen Kunst. Über das Verhalten eigensinniger Objekte. In: K. Ferus / D. Rübel (Hrsg.): „Die Tücke des Objekts“. Vom Umgang mit Dingen, Schriftenreihe der Isa Lohmann-Siems Stiftung 2. Berlin 2009, 129-155.

Rüsen 1990 Rüsen, J.: Die vier Typen des historischen Erzählens. In: J. Rüsen (Hrsg.): Zeit und Sinn. Strategien historischen Denkens. Frankfurt a. M. 1990, 153-230.

Ryan 2007 Ryan, M.-L.: Toward a definition of narrative. In: D. Herman (Hrsg.): The Cambridge companion to narrative. Cambridge / New York 2007, 22-35.

Saupe 2014 Saupe, A.: Authentizität. In: S. Samida / M. K. H. Eggert / H. P. Hahn (Hrsg.): Handbuch Materielle Kultur. Bedeutungen, Konzepte, Disziplinen. Stuttgart 2014, 180-184.

Saupe/Wiedermann 2015 Saupe, A. / Wiedemann, F.: Narration und Narratologie. Erzähltheorien in der Geschichtswissenschaft, Version 1.0, in: Docupedia-Zeitgeschichte, 28.1.2015 <http://docupedia.de/Zg/Narration? oldid $=98435>(01.02 .2015)$.

Schapp 1953 Schapp, W.: In Geschichten verstrickt. Zum Sein von Mensch und Ding. Hamburg 1953.

Schapp 1981 Schapp, W.: Philosophie der Geschichten. Frankfurt a. M. ${ }^{2} 1981$.

Schiffer 1972 Schiffer, M. B.: Archaeological Context and Systemic Context, American Antiquity 37, 1972, 156-165.

Scholtz 2004 Scholtz, G.: Das Verhältnis der Geschichten zur Geschichte. Kritische Fragen an Wilhelm Schapp. In: K.-H. Lembeck (Hrsg.): Geschichte und Geschichten. Studien zur Geschichtenphänomenologie Wilhelm Schapps 7. Würzburg 2004, 57-71.

Schreiber 2013 Schreiber, S.: Archäologie der Aneignung. Zum Umgang mit Dingen aus kulturfremden Kontexten, Forum Kritische Archäologie 2, 2013, 48-123, <http://www.kritischearchaeologie.de/repositorium/ fka/2013_2_05_Schreiber.pdf $>$.

Schreiber im Druck Schreiber, S.: Die Figur der Cyborg in der Vergangenheit. Posthumanismus oder eine neue sozial(er)e Archäologie?, in: Hofmann u. a. im Druck.

Schüttpelz 2013 Schüttpelz, E.: Elemente einer Akteur-Medien-Theorie. In: T. Thielmann / E. Schüttpelz (Hrsg.): Akteur-Medien-Theorie. Bielefeld 2013, 9-70. 
Shanks 2007 Shanks, M.: Symmetrical archaeology, World Archaeology 39, 2007, 589-596.

Sherratt 1995 Sherratt, A.: Reviving the grand narrative. Archaeology and long-term change, Journal of European Archaeology 3, 1995, 1-32.

Stockhammer 2012 Stockhammer, P. W.: Performing the Practice Turn in Archaeology, Transcultural Studies 2012, 7-42.

Strathern 1988 Strathern, M.: The Gender of the Gift. Problems with Women and Problems with Society in Melanesia, Studies in Melanesian anthropology 6. Berkeley 1988.

Straub 2013 Straub, J.: Kann ich mich selbst erzählen - und dabei erkennen? Prinzipien und Perspektiven einer Psychologie des Homo Narrator. In: A. Strohmaier (Hrsg.): Kultur - Wissen - Narration. Perspektiven transdisziplinärer Erzählforschung für die Kulturwissenschaften. Bielefeld 2013, 75-144.

Tringham 1994 Tringham, R.: Engendered places in prehistory, Gender, Place \& Culture. A Journal of Feminist Geography 1, 1994, 169-203.

Tringham 1995 Tringham, R.: Archaeological Houses, Households, Housework and House. In: D. N. Benjamin / D. Stea (Hrsg.): The home. Words, interpretations, meanings and environments. Aldershot / Brookfield / Hong Kong / Singapore / Sidney 1995.

Veit 2006 Veit, U.: Der Archäologe als Erzähler. In: H.-P. Wotzka (Hrsg.): Grundlegungen. Beiträge zur europäischen und afrikanischen Archäologie für Manfred K. H. Eggert. Tübingen 2006, 201-213.

Veit 2010 Veit, U.: Zur Geschichte und Theorie des Erzählens in der Archäologie. Eine Problemskizze, Ethnographisch-Archäologische Zeitschrift 51, 2010, 10-29.

Virchow 1881 Virchow, R.: Vorrede. In: H. Schliemann (Hrsg.): Ilios: Stadt und Land der Trojaner. Forschungen und Entdeckungen in der Troas und besonders auf der Baustelle von Troja. Leipzig 1881, VIII-XIX.

Weiner 1992 Weiner, A. B.: Inalienable possessions. The paradox of keepingwhile-giving. Berkeley 1992.

White 1986 White, H. V.: Auch Klio dichtet, oder, Die Fiktion des Faktischen. Studien zur Tropologie des historischen Diskurses, Sprache und Geschichte 10. Stuttgart 1986.

White 1990 White, H. V.: Die Bedeutung der Form. Erzählstrukturen in der Geschichtsschreibung. Frankfurt a. M. 1990.

Whitley 2002 Whitley, J.: Objects with Attitude. Biographical Facts and Fallacies in the Study of Late Bronze Age and Early Iron Age Warrior Graves, Cambridge Archaeological Journal 12, 2002, 217-232. 
Wiedemann 2010 Wiedemann, F.: Völkerwellen und Kulturbringer. Herkunfts- und Wanderungsnarrative in historisch-archäologischen Interpretationen des Vorderen Orients um 1900, Ethnographisch-Archäologische Zeitschrift 51, 2010, 105-128.

Wiedemann u. a. in Vorbereitung Wiedemann, F. / Hofmann, K. P. / Gehrke, H.-J. (Hrsg.): Vom Wandern der Völker. Migrationserzählungen in den Altertumswissenschaften. Workshop 11./12.10.2012 Berlin, Berlin Studies of the Ancient World (in Vorbereitung).

Witmore 2007 Witmore, C. L.: Symmetrical archaeology: excerpts of a manifesto, World Archaeology 39, 4, 2007, 546-562. 